\title{
Scalable microbial strain inference in metagenomic data using StrainFacts
}

\author{
Byron J. Smith A,B (ORCID: 0000-0002-0182-404X) \\ Xiangpeng $\mathrm{Li}^{\mathrm{C}}$ \\ Adam Abate C,D (ORCID: 0000-0001-9614-4831) \\ Zhou Jason Shi A,D \\ Katherine S. Pollard A,B,D,* (ORCID: 0000-0002-9870-6196) \\ A The Gladstone Institute of Data Science and Biotechnology, San Francisco, CA \\ B Department of Epidemiology and Biostatistics, University of California, San Francisco, CA \\ ${ }^{C}$ Department of Bioengineering and Therapeutic Sciences, University of California, San \\ Francisco, CA \\ D Chan-Zuckerberg Biohub, San Francisco, CA \\ * Corresponding author: \\ katherine.pollard@gladstone.ucsf.edu
}

\section{Abstract}

While genome databases are nearing a complete catalog of species commonly inhabiting the human gut, their representation of intraspecific diversity is lacking for all but the most abundant and frequently studied taxa. Statistical deconvolution of allele frequencies from shotgun metagenomic data into strain genotypes and relative abundances is a promising approach, but existing methods are limited by computational scalability. Here we introduce StrainFacts, a method for strain deconvolution that enables inference across tens of thousands of metagenomes. We harness a "fuzzy" genotype approximation that makes the underlying graphical model fully differentiable, unlike existing methods. This allows parameter estimates to be optimized with gradient-based methods, speeding up model fitting by two orders of magnitude. A graphical processing unit implementation provides additional scalability. Extensive simulations show that StrainFacts can perform strain inference on thousands of metagenomes and has comparable accuracy to more computationally intensive tools. We further validate our strain inferences using single-cell genomic sequencing from a human stool sample. Applying StrainFacts to a collection of more than 10,000 publicly available human stool metagenomes, we quantify patterns of strain diversity, biogeography, and linkage-disequilibrium that agree with and expand on what is known based on existing reference genomes. StrainFacts paves the way for large-scale biogeography and population genetic studies of microbiomes using metagenomic data.

Keywords: metagenomics, strains, microbiome, biogeography, population genetics, modelbased inference 


\section{Introduction}

Intra-specific variation in microbial traits are widespread and are biologically important in human associated microbiomes. Strains of a species may differ in their pathogenicity (1), antibiotic resistance (2), impacts on drug metabolism (3), and ability to utilize dietary components $(4,5)$. Standard methods for analysis of complex microbial communities are limited to coarser taxonomic resolution due to their reliance on slowly evolving marker genes (6) or on genome reference databases lacking diverse strain representation (7). Approaches that quantify microbiomes at the level of strains may better capture variation in microbial function (8), provide insight into ecological and evolutionary processes (9), and discover previously unknown microbial etiologies for disease (10).

Shotgun metagenomic data can in principle be used to track strains by looking for distinct patterns of alleles observed across single nucleotide polymorphisms (SNPs) within the species. Several tools have recently been developed that count the number of metagenomic reads containing alleles across SNP sites (11-16). Comparisons of the resulting "metagenotypes" across samples has been used to track shared strains $(14,17)$, or to interrogate the biogeography $(11,18)$ and population genetics of species $(19)$. The application of this approach is limited, however, by low sequencing coverage, which results in missing values at some SNP sites, and co-existing mixtures of strains, which introduce ambiguity about the taxonomic source of each metagenomic read.

One promising solution to these challenges is statistical strain deconvolution, which harnesses multiple metagenotypes (e.g., a collection of related samples) to simultaneously estimate the genotypes and relative abundances of strains across samples. Several tools have been developed that take this approach, including Lineage (20), Strain Finder (21), DESMAN (22), and ConStrains (23). These methods have been used to track the transmission of inferred strains from donors' to recipients' microbiomes after fecal microbiota transplantation (FMT) $(21,24-26)$. The application of strain deconvolution has been limited, however, by the computational demands of existing methods, where runtimes scale poorly with increasing numbers of samples, latent strains, and SNPs considered. One reason for this poor scaling is the discreteness of alleles at each SNP, which has led existing methods to use expectation maximization algorithms to optimize model parameters (21), or Markov chain Monte Carlo to sample from a posterior distribution $(20,22,23)$.

Here we take a different approach, extending the strain deconvolution framework by relaxing the discreteness constraint and allowing genotypes to vary continuously between alleles. The use of this "fuzzy" genotype approximation makes our underlying model fully differentiable, and allows us to apply modern, gradient-based optimization algorithms to estimate strain genotypes and abundances. Here we show that the resulting tool, StrainFacts, can scale to tens of thousands of samples, hundreds of strains, and thousands of SNPs, opening the door to strain inference in large metagenome collections. 


\title{
Materials and Methods
}

\section{A fully differentiable probabilistic model of metagenotype data}

\author{
Table 1: Symbols used to describe the StrainFacts model \\ Symbols Description \\ $i=1, \ldots, N \quad$ Index and number of samples \\ $s=1, \ldots, S \quad$ Index and number of strains \\ $g=1, \ldots, G \quad$ Index and number of SNP sites \\ $y_{i g}, m_{i g} \quad$ Counts of reads with the alternative allele; the total count of both reference and \\ alternative alleles at SNP $g$ in sample $i$ \\ $p_{i g} \quad$ Alternative allele frequency at SNP $g$ in sample $i$ \\ $\gamma_{s g}, \vec{\gamma}_{g} \quad$ Allele at SNP $g$ in strain $s$; vector of alleles for all strains \\ $\pi_{i s}, \vec{\pi}_{i} \quad$ Relative abundance of strain $s$ in sample $i$; vector of relative abundances for all \\ strains \\ $\epsilon_{i} \quad$ Sequencing error rate in sample $i$ \\ $\alpha \quad$ Concentration parameter of the BetaBinomial distribution \\ $\vec{\rho} \quad$ Metacommunity strain composition \\ $\mathbf{Y}, \mathbf{M}, \mathbf{P}, \mathbf{\Gamma}, \quad$ Matrices composed of the above elements \\ П
}

\section{Metagenotypes}

A metagenotype is represented as a count matrix of the number of reads with each allele at a set of SNP sites for a single species in each sample. This can be gathered directly from metagenomic data, for instance by aligning reads to a reference genome and counting the number of reads with each allele at SNP sites. In this study we use GT-Pro (16), which instead counts exact k-mers associated with known single nucleotide variants. Although the set of variants at a SNP may include any of the four bases, here we constrain metagenotypes to be biallelic: reference or alternative. For a large majority of SNPs, only two alleles are observed across reference genomes (16). Metagenotypes from multiple samples are subsequently combined into a 3-dimensional array.

\section{Deconvolution of metagenotype data}

StrainFacts is based on a generative, graphical model of biallelic metagenotype data (summarized in Fig. 1) which describes the allele frequencies at each SNP site in each sample as the product of the relative abundance of strains and their genotypes. We notate this functional relationship as $p_{i g}=\sum_{s} \gamma_{s g} \times \pi_{i}$, where $\gamma_{s g}$ indicates the allele at SNP $g$ in strain $s$, and equals 1 if it is the alternative allele. In matrix form, equivalently, we notate this as $\mathbf{P}=\Gamma \Pi$ (Table 1).

The crux of strain deconvolution is taking noisy observations of $\mathbf{P}$-based on the observed alternative allele counts $\mathbf{Y}$ and total counts $\mathbf{M}$ obtained from metagenotypes across multiple samples-and determining suitable matrices $\Gamma$ and $\Pi$. This notation highlights parallels to non- 
negative matrix factorization (NMF). Like NMF, given a choice of loss function, $L$, this inference task can be transformed into a constrained optimization problem, where $\arg \min L(\Pi, \Gamma \mid \mathbf{Y})$ is a $\Pi, \Gamma$

scientifically useful estimate of these two unobserved matrices. We take the approach of explicitly modeling the stochasticity of observed metagenotypes, placing priors on $\boldsymbol{\Pi}$ and $\boldsymbol{\Gamma}$, and taking the resulting posterior probability as the loss function. This "maximum a posteriori" (MAP) approach has also been applied to NMF (27). However, unlike NMF, where the key constraint is that all matrices are non-negative, the metagenotype deconvolution model also constrains the elements of $\mathbf{P}$ and $\boldsymbol{\Gamma}$ to lie in the closed interval $[0,1]$ (i.e. the "1-simplex" or $\mathcal{S}^{1}$ for biallelic SNPs), and the rows of $\Pi$ are in $\mathcal{S}^{S-1}$.

\section{Fuzzy genotypes and the shifted-scaled Dirichlet distribution}

StrainFacts does not constrain the elements of $\Gamma$ to be discrete-i.e. in the set $\{0,1\}$ for biallelic sites -in contrast to prior tools: Strain Finder (21), DESMAN (22), and Lineage (20). Instead, we allow genotypes to vary continuously in the open interval between fully reference $(0)$ and fully alternative (1). The use of fuzzy-genotypes serves a key purpose: by replacing the only discrete parameter with a continuous approximation, our posterior function becomes fully differentiable, and therefore amenable to efficient, gradient-based optimization. We show below that inference with StrainFacts is faster than with other tools.

Since true genotypes are in fact discrete, we place a prior on the elements of $\boldsymbol{\Gamma}$ that pushes estimates towards zero or one and away from intermediate-ambiguous—values. Similarly, we put a hierarchical prior on $\Pi$ that regularizes estimates towards lower strain heterogeneity within samples, as well as less strain diversity across samples. This makes strain inferences more parsimonious and interpretable. We harness the same family of probability distributions, the shifted-scaled Dirichlet distribution (SSD) (28), for all three goals. We briefly describe our rationale and parameterization of the SSD distribution in the Supplementary Methods.

For each element of $\Gamma$ we set the prior as $\gamma \sim \operatorname{SSD}_{0}\left(\mathbf{1}, 1, \frac{1}{\gamma^{*}}\right)$. Note that we trivially transform the $\tilde{\gamma} \in \mathcal{S}^{1}$ to the unit interval by dropping the second element. Smaller values of the hyperparameter $\gamma^{*}$ correspond to more sparsity in $\Gamma$. We put a hierarchical prior on $\Pi$, with the rows subject to the prior $\vec{\pi}_{i} \sim \operatorname{SSD}\left(\mathbf{1}, \vec{\rho}, \frac{1}{\pi^{*}}\right)$ given a "metacommunity" hyperprior $\vec{\rho} \sim$ $\operatorname{SSD}\left(1,1, \frac{1}{\rho^{*}}\right)$, reflecting the abundance of strains across all samples. Decreasing the values of $\gamma^{*}, \rho^{*}$, and $\pi^{*}$ increases the strength of regularization imposed by each of these priors.

\section{Model specification}

The underlying allele frequencies $\mathbf{P}$ are not directly observed due to sequencing error, and we include a measurement process in our model. We assume that the true allele is replaced with a random allele at a rate $\epsilon_{i}$ for all SNP sites $g$ in sample $i: \tilde{p}_{i g}=p_{i g}\left(1-\epsilon_{i} / 2\right)+\left(1-p_{i g}\right)\left(\epsilon_{i} / 2\right)$. Given the total counts, $\mathbf{M}$, we then model the observed alternative allele counts, $\mathbf{Y}$, with the BetaBinomial likelihood, parameterized with $\widetilde{\mathbf{P}}$ and one additional parameter- $\alpha^{*}-$ controlling count overdispersion relative to the Binomial model. 
To summarize, our model is as follows (in random variable notation; see Fig. 1 for a plate diagram):

$$
\begin{aligned}
y_{i g} & \sim \operatorname{BetaBinom}\left(\tilde{p}_{i g}, \alpha^{*} \mid m_{i g}\right) \\
\tilde{p}_{i g} & =p_{i g}\left(1-\epsilon_{i} / 2\right)+\left(1-p_{i g}\right)\left(\epsilon_{i} / 2\right) \\
p_{i g} & =\sum_{s} \pi_{i s} \gamma_{s g} \\
\gamma_{s g} & \sim \operatorname{SSD}_{0}\left(\mathbf{1}, \mathbf{1}, \frac{1}{\gamma^{*}}\right) \\
\vec{\pi}_{i} & \sim \operatorname{SSD}\left(\mathbf{1}, \vec{\rho}, \frac{1}{\pi^{*}}\right) \\
\vec{\rho} & \sim \operatorname{SSD}\left(\mathbf{1}, \mathbf{1}, \frac{1}{\rho^{*}}\right) \\
\epsilon & \sim \operatorname{Beta}\left(\epsilon_{a}^{*}, \frac{\epsilon_{a}^{*}}{\epsilon_{b}^{*}}\right)
\end{aligned}
$$

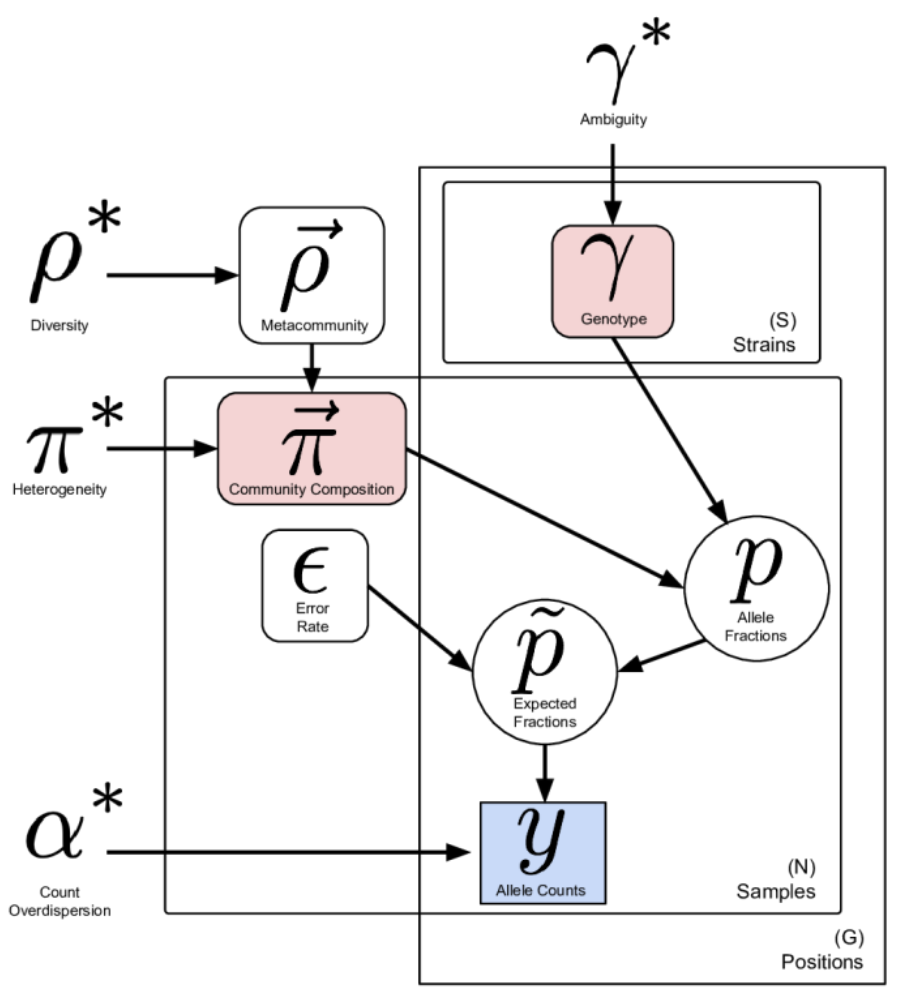

Figure 1: Graphical representation of the StrainFacts model including hyperparameters. Symbols include observed data (blue box), deterministic terms (circles), key parameters being estimated (red boxes), and key hyperparameters (unenclosed). Plates behind terms indicate the dimensionality and indexing of the variables and arrows connect the terms that directly depend on one another. The distribution family and relationships between terms are indicated on the right.

\section{Model fitting}

StrainFacts takes a MAP-based approach to inference on this model, using gradient-based methods to find parameter values that maximize the posterior probability of our model conditioned on the observed counts. We rely heavily on the probabilistic programming framework Pyro (29), which is built on the PyTorch library (30) for numerical methods.

Initial values for $\boldsymbol{\Gamma}$ and $\boldsymbol{\Pi}$ are selected using NMF, and all other parameters are initialized randomly (Supplementary Methods). In order to promote global convergence, we take a prior annealing approach (Supplementary Methods). While it is impossible to know in practice if we converge to a global optimum, we find that this procedure often leads to accurate estimates without the need for replicate fits from independent initializations.

\section{Simulation and benchmarking}

Metagenotype data was simulated in order to enable direct performance benchmarking against ground-truth genotypes and strain compositions. For each independent simulation, discrete genotypes of length $G$ for $S$ strains were sampled as $S \times G$ independent draws from a symmetric Bernoulli distribution. The composition of strains in each of $N$ samples were generated as independent draws from a Dirichlet distribution over $S$ components having a symmetric 
concentration parameter of 0.4 . Per-sample allele frequencies were generated as the product of the genotypes and the strain-composition matrices. Sequence error was set to $\epsilon=0.01$ for all samples. Finally metagenotypes at each SNP site were drawn from a $\operatorname{Binomial}\left(m, \tilde{p}_{i g}\right)$ distribution, with a sequencing depth of $m=10$ across all sites.

Estimates were evaluated against the simulated ground truth using five different measures of error (see Results).

\section{Metagenotypes and reference genomes}

We applied StrainFacts to data from two previously compiled human microbiome metagenomic datasets: stool samples from a fecal microbiota transplantation (FMT) study described in (26) and 20,550 metagenomes from a meta-analysis of publicly available data in (16). As described in that publication, metagenotypes for gut prokaryotic species were tallied using GT-Pro version 1.0.1 with the default database, which includes up to 1000 of the highest quality genomes for each species from the Unified Human Gastrointestinal Genome (UHGG) V1.0 (31). This includes both cultured isolates and high-quality metagenomic assemblies. This same database was used as a reference set to which we compared our inferred genotypes. The five species for which we describe detailed results are: Streptococcus thermophilus (GT-Pro species id: 104345, representative UHGG genome: MGYG-HGUT-04345), Escherichia coli_D (id: 102506, MGYG-HGUT-02506), Agathobacter rectalis (id: 102492, MGYG-HGUT-02492), Methanobrevibacter_A smithii (id: 102163, MGYG-HGUT-02163), and CAG-279 sp1 (id: 102556, MGYG-HGUT-02556). Estimated genomic distances between SNPs were based on the UHGG representative genome.

\section{Single-cell genome sequencing}

A full description of the single-cell genomics pipeline is included in the Supplementary Methods, and will be briefly summarized here. For one of the 159 samples with metagenomes described in the FMT study, microbial cells were isolated from whole feces by homogenization in phosphate buffered saline, $50 \mu \mathrm{m}$ filter-based removal of large fecal particles, and density gradient separation. After isolating and thoroughly washing the density layer corresponding to the microbiota, this cell suspension was mixed with polyacrylamide precursor solution, and emulsified with a hydrofluoric oil. Aqueous droplets in oil were allowed to gellate before separating the resulting beads from the oil phase and washing. Beads were size selected to between 5 and $25 \mu \mathrm{m}$, with the goal of enriching for those encapsulated a single microbial cell. Cell lysis was carried out inside the hydrogel beads by incubating with zymolyase, lysostaphin, mutanolysin, and lysozyme. After lysis, proteins were digested with proteinase $\mathrm{K}$, before thoroughly washing the beads. Tn5 tagmentation and barcode PCR were carried out using the MissionBio Tapestri microfluidics DNA workflow with minor modifications. After amplification, the emulsion was broken and the aqueous phase containing the barcoded amplicons was used for sequencing library preparation with Nextera primers including P5 and P7 sequences followed by Ampure XP bead purification. Libraries were sequenced by Novogene on an Illumina NovaSeq 6000.

Demultiplexed sequence data for each droplet barcode were independently processed with GTPro identically to metagenomic sequences. For each barcode, GT-Pro allele counts for a given species were assumed to be representative of a single strain of that species. These single-cell genotypes (SCGs) were filtered to those with $>1 \%$ horizontal coverage over SNP sites, leaving 87 species with at least one SCG from either of the two focal samples. During analysis, a number of SCGs were found to have nearly identical patterns of horizontal coverage. These may have been formed by merging of droplets during barcoding PCR, which could have 
resulted in multiple barcodes in the same amplification. To reduce the impact of this artifact, allele counts from multiple SCGs were summed by complete-linkage, agglomerative clustering based on their depth profiles across SNP sites, at a 0.3 cosine dissimilarity threshold.

\section{Computational Analysis}

\section{Metagenotype filtering}

From GT-Pro metagenotypes, we extracted allele counts for select species and removed SNPs that had $<5 \%$ occurance of the minor allele across samples. Species with more than 5,000 SNPs after filtering, were randomly down-sampled without replacement to this number of sites. Samples with less than a minimum horizontal coverage (fraction of SNP sites with non-zero total counts), were also filtered out. This horizontal coverage threshold was set to $5 \%$ or $25 \%$ for the datasets from (26) or (16), respectively.

\section{Strain Inference}

For all analyses, StrainFacts was run with the following hyperparameters $\rho^{*}=0.5, \pi^{*}=0.3$, $\gamma^{*}=10^{-10}, \alpha^{*}=10, \epsilon_{a}^{*}=1.5, \epsilon_{b}^{*}=0.01$. The learning rate was initially set to 0.05 . Prior annealing was applied to both $\Gamma$ and $\vec{\rho}$ by setting $\gamma^{*}$ and $\rho^{*}$ to 1.0 and 5 , respectively, for the first 2,000 steps, before exponentially relaxing these hyperparameters to their final values over the next 8,000 steps. After this annealing period, when parameters had not improved for 100 steps, the learning rate was halved until it had fallen below 10-6, at which point we considered parameters to have converged.

The number of strains parameterized by our model was chosen as follows. For comparisons to SCGs, the number of strains was set at $30 \%$ of the number of samples-e.g. 33 strains were parameterized for $S$. thermophilus because metagenotypes from 109 samples remained after coverage filtering. For the analysis of thousands of samples described in (16), we parameterized our model with 200 strains and increased the numerical precision from 32 to 64 bits. After strain inference using the 5,000 subsampled SNPs, full-length genotypes were estimated post-hoc by conditioning on our estimate of $\Pi$ and iteratively refitting subsets of all SNPs (Supplementary Methods).

For computational reproducibility we set fixed seeds for random number generators: 0 for all analyses where we only report one estimate, and $0,1,2,3$, and 4 for the five replicate estimates described for simulated datasets. Strain Finder was not originally designed to take a random seed argument, necessitating minor modifications to the code.

\section{Genotype comparisons}

Inferred fuzzy genotypes were discretized to zero or one for downstream analyses. Similarly, when comparing genotypes to the metagenotype consensus or SCGs, observed allele frequencies were discretized to the majority allele. SNP sites without coverage were treated as unobserved. Distances between genotypes were calculated as the masked, normalized Hamming distance, the fraction of alleles that do not agree, ignoring unobserved SNP. Metagenotype entropy, a proxy for strain heterogeneity, was calculated for each sample as the depth weighted mean allele frequency entropy: $\frac{1}{\sum_{g} m_{i g}} \sum_{g}-m_{i g}\left[\left(\hat{p}_{i g} \log _{2}\left(\hat{p}_{i g}\right)+\right.\right.$ $\left.\left(1-\hat{p}_{i g}\right) \log _{2}\left(1-\hat{p}_{i g}\right)\right]$ where $\hat{p}_{i g}$ is the observed alternative allele frequency.

Where indicated, we dereplicated highly similar strains by applying average-neighbor agglomerative clustering at a 0.05 genotype distance threshold. Groups of these highly similar 
strains were replaced with a single composite strain with a genotype derived from the majority allele at each SNP site and assigned the sum of strain relative abundances in each sample. Subsequent co-clustering of these dereplicated inferred and reference strains was done in the same way, but at a 0.15 genotype distance threshold. After co-clustering, to test for enrichment of strains in "shared" clusters, we permuted cluster labels and re-tallied the total number of strains found in clusters with both inferred and reference strains. Likewise, to test for enrichment of "inferred-only" clusters we tallied the total number of strains found in clusters without reference strains after this shuffling. By repeating the permutation 9999 times, we arrived at an empirical null distribution to which we compared our true, observed values to calculate a Pvalue.

Pairwise linkage disequilibrium (LD) was calculated as the squared Pearson correlation coefficient across genotypes of dereplicated strains. To calculate the 90th percentile LD, SNP pairs were binned at either an exact genomic distance or within a window of distances, as indicated. In order to encourage a smooth distance-LD relationship, windows at larger pairwisedistance spanned a larger range. Specifically the ith window covers the span $\left[\left\lfloor 10^{(i-1) / c}\right\rfloor,\left\lfloor 10^{i / c}\right\rfloor\right)$ where $c=30$ so that 120 windows span the full range $\left[1,10^{4}\right)$.

\section{Software and code availability}

StrainFacts is implemented in Python 3 and is available at https://github.com/bsmith89/StrainFacts and v0.1 was used for all results reported here. A forked version of Strain Finder-modified for ease of installation and to specify a random seed for reproducibility—can be found at https://github.com/bsmith89/StrainFinder. All other code and metadata needed to re-run these analyses is available at https://github.com/bsmith89/StrainFacts-manuscript. For software reproducibility, all analyses were performed using a Singularity container (32).

\section{Results}

\section{Scaling strain inference to hundreds of genotypes in thousands of samples}

Inferring the genotypes and relative abundance of strains in large metagenome databases requires a deconvolution tool that can scale to metagenotypes with thousands of SNPs in tensof-thousands of samples, while simultaneously tracking hundreds of microbial strains. To accomplish this we developed StrainFacts, harnessing fuzzy genotypes to accelerate inference on large datasets. We evaluated the practical scalability of the StrainFacts algorithm by applying it to simulated datasets of increasing size, and comparing its time and memory requirements to Strain Finder, a previously described method for strain inference. While several tools have been developed to perform strain deconvolution (e.g. Lineage 20, and DESMAN 22), Strain Finder's model and approach to inference are the most similar to StrainFacts. We therefore selected it for comparison in order to directly assess the value of fuzzy genotypes.

We simulated five replicate metagenotypes for 120 underlying strains in 400 samples, and 250 SNPs, and then applied both StrainFacts and Strain Finder to these data parameterizing them with 120 strains. Both tools use random initializations, which can result in convergence to different optima. We therefore benchmarked runtimes for five independent initializations on each dataset, resulting in 25 total runs for each tool. In this setting, the median runtime for StrainFacts was just 17.2 minutes, while Strain Finder required a median of 6.4 hours. When 
run on a GPU instead of CPU, StrainFacts was able to fit these data in a median of just 5.1 minutes.

Since the correct strain number is not known a priori in real-world applications, existing strain inference tools need to be parameterized across a range of plausible strain counts, a step that significantly impacts runtime. To assess performance in this setting, we also fit versions of each model with $50 \%$ more strains than the ground-truth, here referred to as the " $1.5 \mathrm{x}$ parameterization" in contrast to the 1x parameterization already described. In this setting, StrainFacts' performance advantage was even more pronounced, running in a median of 17.1 minutes and just 5.3 minutes on GPU, while Strain Finder required 30.8 hours. Given the speed of StrainFacts, we were able to fit an even larger simulation with 2,500 samples and 500 strains. On a GPU, this took a median of 12.6 minutes with the $1 x$ parameterization and, surprisingly, just 8.9 minutes with the $1.5 x$ parameterization. We did not attempt to run Strain Finder on this dataset.

We next examined runtime scaling across a range of sample counts between 50 and 2,500. We applied Strain Finder and StrainFacts (CPU and GPU) to simulated metagenotypes with 250 SNPs, and a fixed 1:5 ratio of strains to samples. Median runtimes for each tool at both the $1 \mathrm{x}$ and $1.5 x$ parameterization demonstrate a substantially slower increase for StrainFacts as model size increases (Fig. 2A). Strain Finder was faster than StrainFacts on the 1x parameterization of a small simulation with 50 samples and 10 strains: 1.3 minutes median runtime versus 4 minutes for StrainFacts on a CPU and 2.8 minutes on a GPU. However, StrainFacts had faster median runtimes on all other datasets.

A

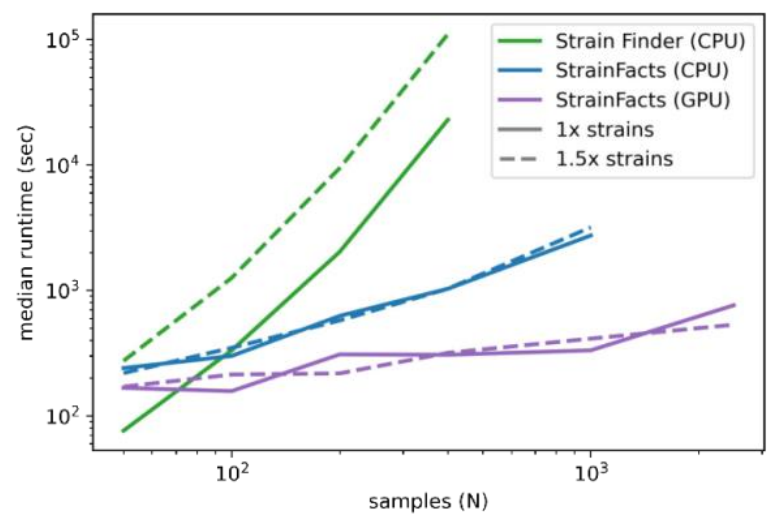

B

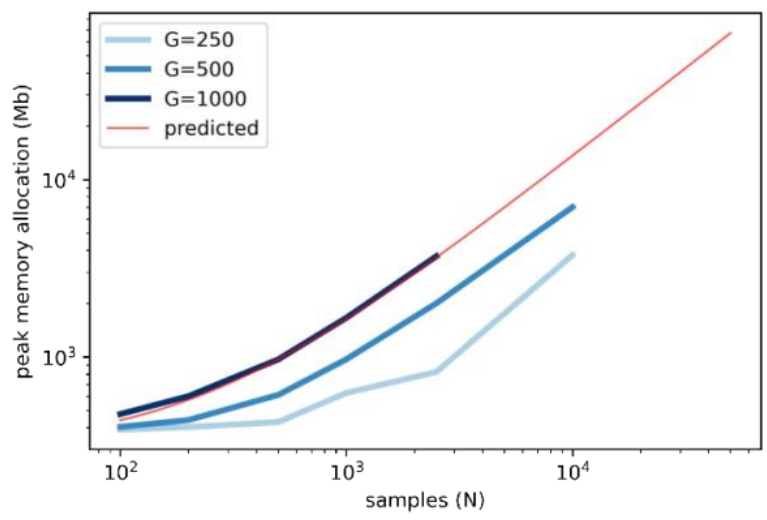

Figure 2: Computational scalability of strain inference on simulated data. (A) Runtime (in seconds, log scale) is plotted at a range of sample counts for both Strain Finder and StrainFacts, as well for the latter with GPU acceleration. Throughout, 250 SNPs are considered, and simulated strains are fixed at a 1:5 ratio with samples. Models are specified with this same number of strains ("1x strains", solid lines) or $50 \%$ more ("1.5x strains", dashed lines). Median of 25 simulation runs is shown. (B) Maximum memory allocation in a model with 100 strains is plotted for StrainFacts models across a range of sample counts $(N)$ and SNP counts (G, line shade). Median of 9 replicate runs is shown. Maximum memory requirements are extrapolated to higher numbers of samples for a model with 1000 SNPs (red line). A version of this panel that includes a range of strain counts is included as Supplementary Fig. S1.

Given the good runtime scaling properties of StrainFacts, we next asked if computer memory constraints would limit its applicability to the largest datasets (Fig. 2A). A model fitting 10,000 samples, 400 strains, and 500 SNPs had a maximum memory allocation of 7.7 GB, indicating that StrainFacts' memory requirements are satisfied on most contemporary CPU or GPU hardware and opening the door to even larger models. Using ordinary least squares, we fit the 
observed memory requirements to the theoretical, asymptomatic expectations, $\mathcal{O}(N S+N G+S G)$, resulting in a regression $\mathrm{R}^{2}$ of 0.997 . We then used this empirical relationship to extrapolate for even larger models (Fig. 2B), estimating that for a model of 400 strains and 1000 SNPs, 32 GB of memory would be able to simultaneously perform strain inference for more than 22,000 samples. This means StrainFacts can realistically analyse tens of thousands of samples on commercial GPUs.

\section{StrainFacts accurately reconstructs genotypes and population structure}

We next set out to evaluate the accuracy of StrainFacts and to compare it to Strain Finder. We simulated 250 SNPs for 40 strains, generating metagenotypes across 200 samples. For both tools, we specified a model with the true number of strains, fit the model to this data, and compared inferences to the simulated ground-truth. For each of five replicate simulations we performed inference with five independent initializations, thereby gathering 25 inferences for each tool. As in (21), we use the weighted UniFrac distance (33) as an integrated summary of both genotype and relative abundance error. By this index, StrainFacts and Strain Finder performed similarly well when applied to the simulated data (Fig. 3A). We repeated this analysis with the $1.5 x$ parameterization to assess the robustness of inferences to model misspecification, finding that both tools maintained similar performance to the $1 \times$ parameterization. Thus, we conclude based on UniFrac distance that StrainFacts is as accurate as Strain Finder and that both models are robust to specifying too many strains.

To further probe accuracy, we quantified the performance of StrainFacts and Strain Finder with several other measures. First, we evaluated pairwise comparisons of strain composition by calculating the mean absolute error of pairwise Bray-Curtis dissimilarities (Fig. 3B). While Strain Finder slightly outperformed StrainFacts on this index under the 1.5x parameterization, the magnitude of the difference was small. This suggests that StrainFacts can be used for applications in microbial ecology that rely on measurements of beta diversity.

Ideally, inferences should conform to Occam's razor, estimating "as few strains as possible, but no fewer". Unfortunately, Bray-Curtis error is not sensitive to the splitting or merging of coabundant strains and UniFrac error is not sensitive to the splitting or merging of strains with very similar genotypes. To overcome this limitation, we calculated the mean absolute error of the Shannon entropy of the inferred strain composition for each sample (Fig. 3C). This score quantifies how accurately inferences reflect within-sample strain heterogeneity. StrainFacts performed substantially better on this score than Strain Finder, both for the $1 \mathrm{x}$ and $1.5 \mathrm{x}$ parameterization, indicating more accurate estimation of strain heterogeneity.

Finally, we assessed the quality of genotypes reconstructed by StrainFacts compared to Strain Finder using the abundance weighted mean Hamming distance. For each ground-truth genotype, normalized Hamming distance is computed based on the best matching inferred genotype (Fig. 3D). We assessed the reverse as well: the abundance weighted mean, bestmatch Hamming distance for each inferred genotype among the ground-truth genotypes (Fig. 3E). These two scores can be interpreted as answers to the distinct questions "how well were the true genotypes recovered?" and "how well do the inferred genotypes reflect the truth?", respectively. While StrainFacts and Strain Finder performed similarly on these indexes-which tool had higher accuracy varied by score and parameterization-StrainFacts' accuracy was more stable between the two parameterizations. 
A

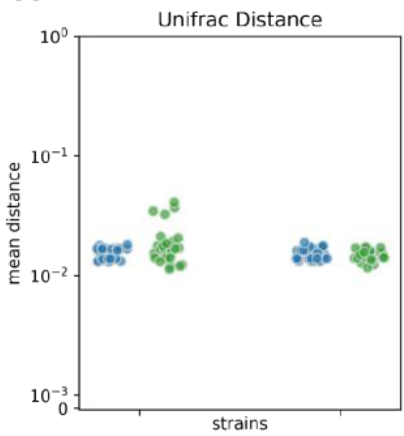

D

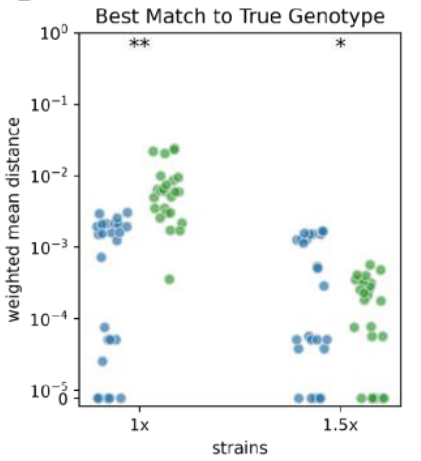

B

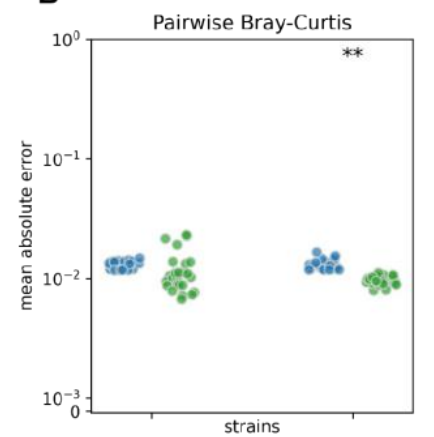

E

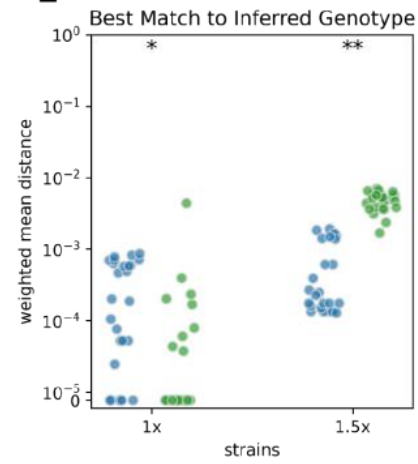

C

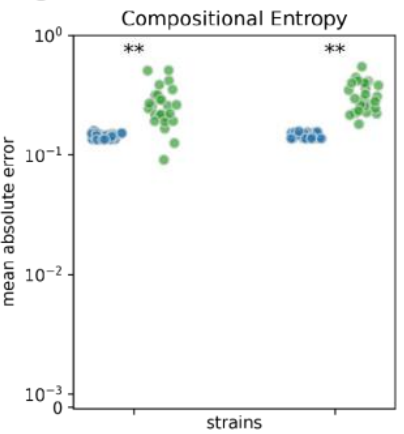

StrainFacts Strain Finder

Figure 3: Accuracy of strain inference on simulated data. Performance of StrainFacts and Strain Finder are compared across five distinct accuracy indices, with lower scores reflecting better performance on each index. Simulated data had 200 samples, 40 underlying strains, and 250 SNPs. For each tool, both 40 and 60 strain models were parameterized (" $1 x$ " and " $1.5 x$ " respectively), and every model was fit with five independent initializations to each simulation. All 25 estimates for each tool-parameterization combination are shown. Scores reflect $(\boldsymbol{A})$ mean Unifrac distance between simulated and inferred strain compositions, (B) mean absolute difference between all-by-all pairwise Bray-Curtis dissimilarities calculated on simulated versus inferred strain compositions, (C) mean absolute difference in Shannon entropy calculated on simulated versus inferred strain compositions, (D) abundance weighted mean Hamming distance from each ground-truth strain to its best-match inferred genotype, and $(\boldsymbol{E})$ the reverse: abundance weighted mean Hamming distance from each inferred strain to its best-match true genotype. Markers at the top of each panel indicate a statistical difference between tools at a $p<0.05\left(^{*}\right)$ or $p<0.001$ $\left.{ }^{* *}\right)$ significance threshold by Wilcoxon signed-rank test.

Overall, these results suggest that StrainFacts is capable of state-of-the-art performance with respect to several different scientific objectives in a realistic set of simulations. Performance was surprisingly robust to model misspecification with $50 \%$ more strains than were simulated.

Eliminating the computational demands of a separate model selection step further improves the scaling properties of StrainFacts.

\section{Single-cell sequencing validates inferred strain genotypes}

Beyond simulations, we sought to confirm the accuracy of strain inferences in a real biological dataset subject to forms of noise and bias not reflected in the generative model we used for simulations. To accomplish this, we applied a recently developed, single-cell, genomic sequencing workflow to obtain ground-truth, strain genotypes from a human fecal sample collected in a previously described, clinical FMT experiment (26). We ran StrainFacts on metagenotypes derived from this and other samples in the same study. 
Genotypes that StrainFacts inferred to be present in the metagenome matched those observed SCGs, with a mean, best-match normalized Hamming distance of 0.049 (Fig. 4A). Furthermore, the median distance was just 0.015 , reflecting the outsized influence of a small number of SCGs with more extreme deviations. For many species, SCGs also match a consensus genotype-the majority allele at each SNP site in the metagenotype, although this comparison is made more challenging by the sparsity of consensus genotypes (Supplementary Fig. S2). Overall, we found a mean distance to the consensus of 0.056 and a median of 0.013 , not significantly different from inferred genotypes ( $p=0.3$ by Wilcoxon signed-rank test). However, the consensus approach fails for species with a mixture of multiple, co-existing strains. When we select only species with a metagenotype entropy of greater than 0.25 , an indication of strain heterogeneity, we see that StrainFacts inferences have a distinct advantage, with a mean distance of 0.115 versus 0.163 for the consensus approach (median of 0.093 versus $0.151, p=0.005$ ). These results validate inferred genotypes in a stool microbiome using single-cell genomics and demonstrate that StrainFacts accounts for strain-mixtures better than consensus genotypes do.

A

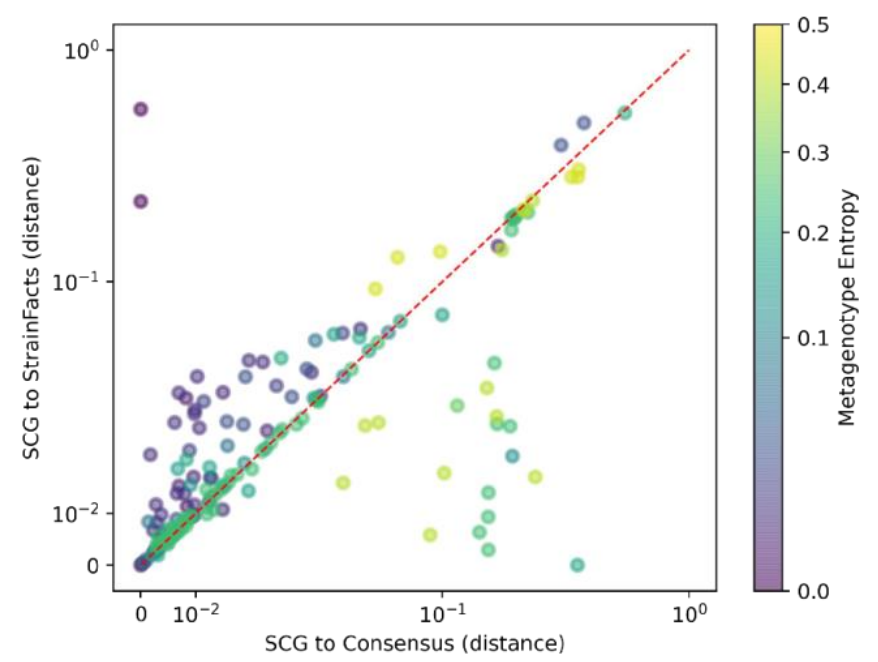

B

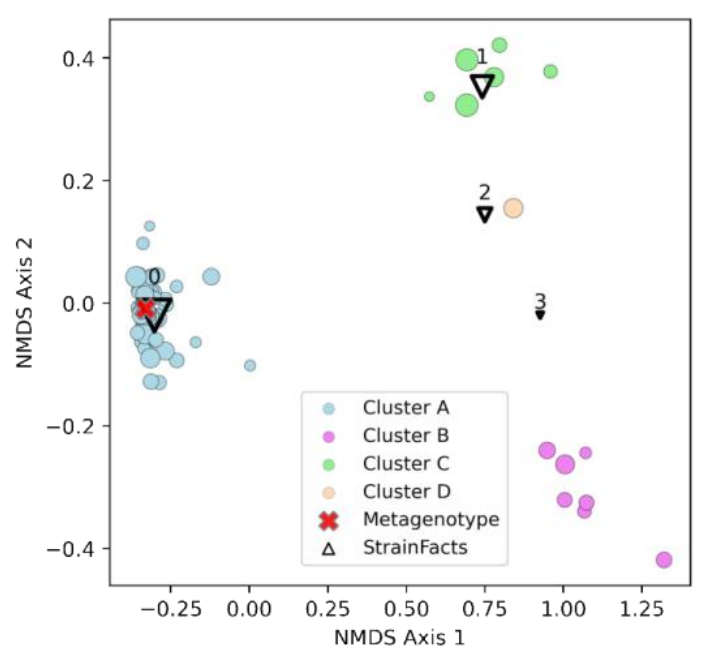

Figure 4: Inferred strains reflect genotypes from a single-cell sequencing experiment. (A) Distance between observed SCGs and StrainFacts inferences ( $X$-axis) versus consensus genotypes ( $Y$-axis). Points below and to the right of the red dotted line reflecting an improvement of our method over the consensus, based on the normalized, best-match Hamming distance. Each dot represents an individual SCG reflecting a putative genotype found in the analysed sample. SCGs from all species found in one sample are represented, and marker colors reflect the metagenotype entropy of that species, a proxy for the potential strain diversity represented. Axes are on a "symmetric" log scale, with linear placement of values below 10-2. A version of this panel colored by metagenotype horizontal coverage is in Supplementary Fig. S2. (B) A non-metric multidimensional scaling ordination of 68 SCGs and inferred genotypes for one species, $S$. thermophilus, with notably high strain diversity in the focal sample. Circles represent SCGs, are colored by their assignment to one of four identified clusters, and larger markers indicate greater horizontal coverage. Triangles represent StrainFacts genotypes inferred to be at greater than $1 \%$ relative abundance, and larger markers reflect a higher inferred relative abundance. The red cross represents the consensus metagenotype of the analysed sample.

Of the 75 species represented in our SCG dataset, one stood out for having numerous SCGs while reflecting a remarkably high degree of strain heterogeneity. Among 68 high-quality SCGs for $S$. thermophilus, cluster analysis identified four distinct types (here referred to as Clusters A D), accounting for 48, 7, 6, and 1 SCGs, respectively (Fig. 4B). Independently, StrainFacts inferred four strains in the metagenomic data from the same stool sample, (Strain 1 - 4) with $57 \%, 32 \%$, and $7 \%$, and $3 \%$ relative abundance, respectively. We explored the concordance 
between clusters and StrainFacts inferences by assigning a best-match Hamming distance genotype among the inferred strains to each SCG (Table 2). For SCGs in three of the four clusters there was a low median distance to StrainFacts genotypes as well as a perfect 1-to-1 correspondence between strains and clusters. While this genotype concordance was broken for SCGs in cluster B, strain 4 was also inferred to be at the lowest relative abundance, suggesting that there may have been too little information encoded in the metagenotype data to accurately reconstruct that strain's genotype. While SCG counts and inferred strain fractions do not match perfectly in this sample, this may be due to large differences between SCG and metagenomic sequencing technologies that could result in differentially biased sampling of strains. The SCG cluster with the largest membership was, however, matched with the strain inferred to be at the highest relative abundance. Our findings for $S$. thermophilus show that StrainFacts' estimates of genotypes and relative abundances are remarkably accurate for samples with high strain heterogeneity, despite the challenges presented by real biological samples and low abundance strains.

Table 2: Concordance among SCGs of cluster assignments and the closest-match StrainFacts inferred genotype, among the four strains inferred to be at greater than $1 \%$ relative abundance in the analysed sample. The total number of SCGs in each cluster and the relative abundance of each inferred strain are indicated in parentheses in the column and row labels, respectively. Numbers in each cell indicate the number of SCGs at that intersection and values in parentheses indicate the median normalized Hamming distance of those SCGs to the inferred strain genotype.

$\begin{array}{lllll} & \text { Cluster A (48) } & \text { Cluster B (7) } & \text { Cluster C (6) } & \text { Cluster D (1) } \\ \text { Strain 1 (57\%) } & 48(0.006) & 1(0.18) & & \\ \text { Strain 2 (32\%) } & 3(0.19) & 6(0.008) & 1(0.02) \\ \text { Strain 3 (7\%) } & 3(0.19) & & \\ \text { Strain 4 (3\%) } & & & \end{array}$

\section{Analysis of genomic diversity using de novo strain inferences on thousands of samples}

Having established the accuracy and scalability of StrainFacts, we applied it to a corpus of metagenotype data derived from 20,550 metagenomes across 44 studies, covering a large fraction of all publicly available human-associated microbial metagenomes (16). We performed strain inference on GT-Pro metagenotypes for four species: Escherichia coli, Agathobacter rectalis, Methanobrevibacter smithii, and CAG-279 sp1. E. coli and A. rectalis are two highly prevalent and well studied bacterial inhabitants of the human gut microbiome, and $M$. smithii is the most prevalent and abundant archaeon detected in the human gut (34). CAG-279, on the other hand, is an unnamed and little-studied genus and a member of the family Muribaculaceae. This family is common in mice (35), but to our knowledge does not have representatives cultured from human samples.

For each species, we compared strains inferred by StrainFacts to those represented in the GTPro reference database, which is derived from the UHGG (31). In order to standardize comparisons, we dereplicated inferred and reference strains at a 0.05 genotype distance threshold. Interestingly, dereplication had a negligible effect on StrainFacts results, reducing the number of $E$. coli strains by just 4 (to 119) with no reduction for the three other species. This 
suggests that the diversity regularization built into the StrainFacts model is sufficient to collapse closely related strains as part of inference.

\section{StrainFacts recapitulates known diversity in well studied species}

E. coli, A. rectalis, and M. smithii all have many genome sequences in GT-Pro reference database, presenting an opportunity to contrast inferred against reference strains. In order to evaluate the concordance between the two (Table 3 and Fig. 5), we co-clustered all dereplicated strains (both reference and inferred) at a 0.15 normalized Hamming distance threshold-note, crucially, that this distance reflects a much smaller full-genome dissimilarity, as it is based only on genome positions with polymorphism across metagenomes, ignoring conserved positions.

For E. coli, we identified 40 strain clusters with $93 \%$ of inferred strains and $94 \%$ of references falling into clusters containing strains from both sources ("shared" clusters), which is significantly more overlap than expected after random shuffling of cluster labels $(p=0.002$ by permutation test). While most metagenome-inferred genotypes are similar to those found in genome reference databases, we observed some clusters composed only of StrainFacts strains, representing novel lineages. However, these strains are no more common than after random permutation ( $p=0.81$ ), matching our expectations for this well-studied species.

We next asked if these trends hold for the other species. While $A$. rectalis had a much greater number of clusters (456), $69 \%$ of inferred strains and $45 \%$ of reference strains are nonetheless found to be in shared clusters, significantly more than would be expected with random shuffling of cluster labels ( $p=0.002$ by permutation test). Correspondingly, we do not find evidence for enrichment of inferred strains in novel clusters $(p=0.71)$. We find similar results for $M$. smithii and CAG-279 - the fraction of strains in shared clusters is significantly greater than after random reassignment ( $p<0.001$ for both), and there is no evidence for enrichment of inferred strains in novel clusters ( $p=1.0$ for both). Overall, the concordance between reference and inferred strains supports not only the credibility of StrainFacts' estimates, but also suggests that our de novo inferences capture a substantial fraction of previously documented strain diversity, even in well studied species.

Table 3: Dereplication and co-clustering of strains inferred from metagenomes or from a reference database

$\begin{array}{lllllll}\text { Species } & \begin{array}{l}\text { Metagenome } \\ \text { samples fit }\end{array} & \begin{array}{l}\text { Reference } \\ \text { strains }^{\mathrm{a}}\end{array} & \begin{array}{l}\text { Inferred } \\ \text { strains }^{\mathrm{a}}\end{array} & \begin{array}{l}\text { Total } \\ \text { Clusters }^{b}\end{array} & \begin{array}{l}\text { Novel } \\ \text { Clusters }^{b}\end{array} & \begin{array}{l}\text { Shared } \\ \text { Clusters }^{b}\end{array} \\ \text { E. coli } & 9232 & 176 & 119 & 40 & 20 \% & 60 \% \\ \text { A. rectalis } & 11860 & 752 & 198 & 456 & 13 \% & 25 \% \\ \text { M. smithii } & 3528 & 384 & 178 & 205 & 7 \% & 38 \% \\ \text { CAG-279 } & 3579 & 135 & 200 & 228 & 50 \% & 25 \%\end{array}$

${ }^{a}$ Dereplicated at 0.05 distance threshold

${ }^{b}$ Co-clustered at a 0.15 distance threshold

Going beyond the extensive overlap of strains with reference genomes and StrainFacts inferences, we examined clusters in which references are absent or relatively rare. Visualizing a dendrogram of consensus genotypes from co-clustered strains (Fig. 5) we observe some sections of the $A$. rectalis dendrogram with many novel strains. Similarly, for CAG-279, the sheer number of inferred strains relative to genomes in reference databases means that fully 
bioRxiv preprint doi: https://doi.org/10.1101/2022.02.01.478746; this version posted February 4, 2022. The copyright holder for this preprint (which was not certified by peer review) is the author/funder, who has granted bioRxiv a license to display the preprint in perpetuity. It is made available under aCC-BY 4.0 International license.

half of all genotype clusters are entirely novel, emphasizing the power of StrainFacts inferences in understudied species. Future work will be needed to determine if these represent new subspecies currently missing from reference databases.

E. coli

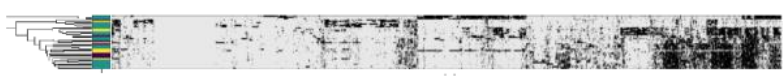

A. rectalis

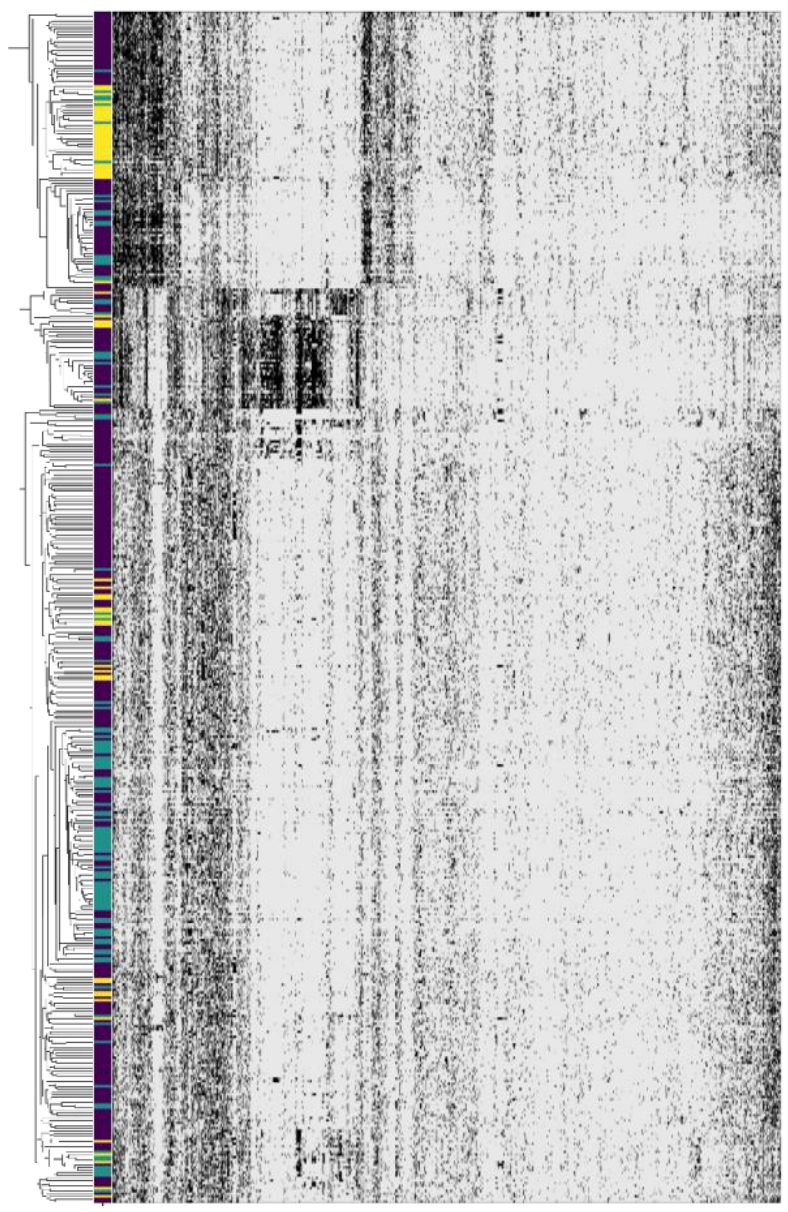

M. smithii

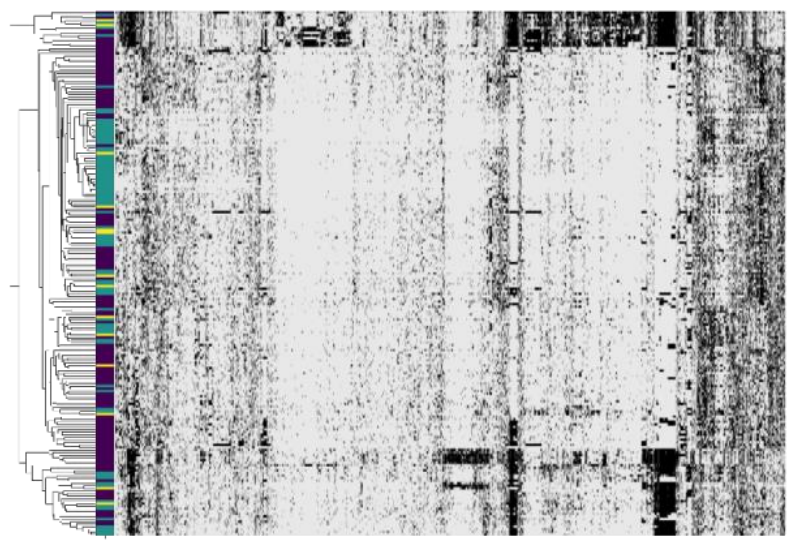

- Reference Both $\quad$ Inferred

CAG-279

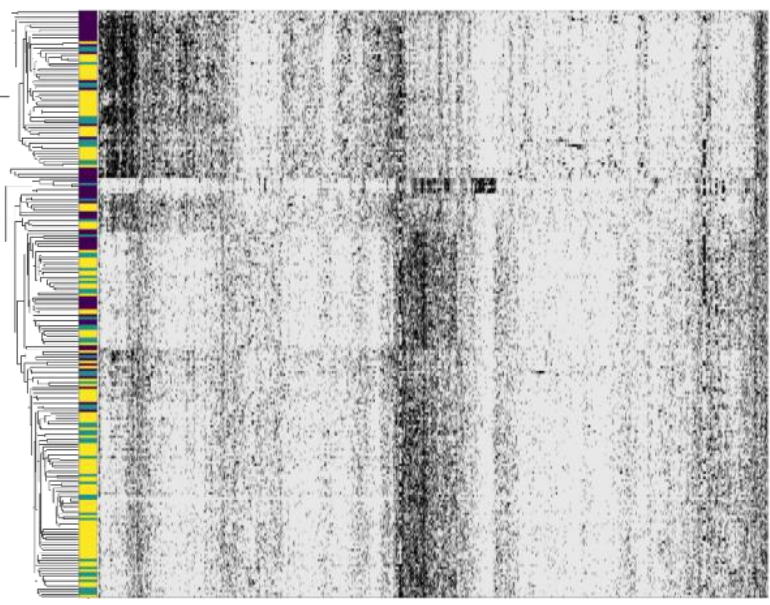

Figure 5: Concordance between reference and StrainFacts inferred strain genotypes for four prevalent species in the human gut microbiome. Heatmap rows represent consensus genotypes from co-clustering of reference and inferred strains and columns are 3500 randomly sampled SNP sites (grey: reference and black: alternative allele). Colors to the left of the heatmap indicate clusters with only reference strains (dark purple), only inferred strains (yellow), or both (teal). Rows are ordered by hierarchical clustering built on distances between consensus genotypes and columns are ordered arbitrarily to highlight correlations between SNPS.

\section{Species inhabiting the human gut exhibit distinct biogeography observed across independent metagenomic studies}

Large metagenomic collections allow us to examine intraspecific microbial diversity at a global scale and among dozens of studies. Towards this end, we identified the most abundant StrainFacts strain of E. coli, A. rectalis, M. smithii, and CAG-279 in stool samples across 34 independent studies. Across all four species, we observe some strains that are distributed 
bioRxiv preprint doi: https://doi.org/10.1101/2022.02.01.478746; this version posted February 4, 2022. The copyright holder for this preprint (which was not certified by peer review) is the author/funder, who has granted bioRxiv a license to display the preprint in perpetuity. It is made available under aCC-BY 4.0 International license.

globally as well as others that appear specific to one country of origin (Fig. 6, Supplementary Fig. S3). For example, among the 198 dereplicated, inferred strains of $A$. rectalis, 75 were found as the dominant strain (i.e. highest relative abundance) in samples collected on three or more continents. While this makes it challenging to consistently discern where a sample was collected based on its dominant strain of a given species, we nonetheless find that studies with samples collected in the United States of America form a distinct cluster, as do those from China, and the two are easily distinguished from one another and from most other studies conducted across Europe and North America (Fig. 6A). Our observation of a distinct group of $A$. rectalis strains enriched in samples from China is consistent with previous results $(11,18,36)$.

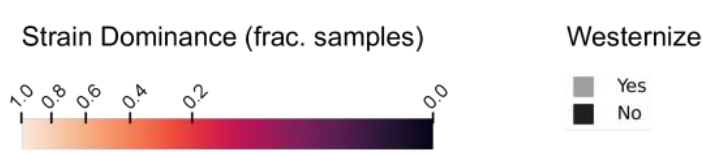

A. rectalis

A

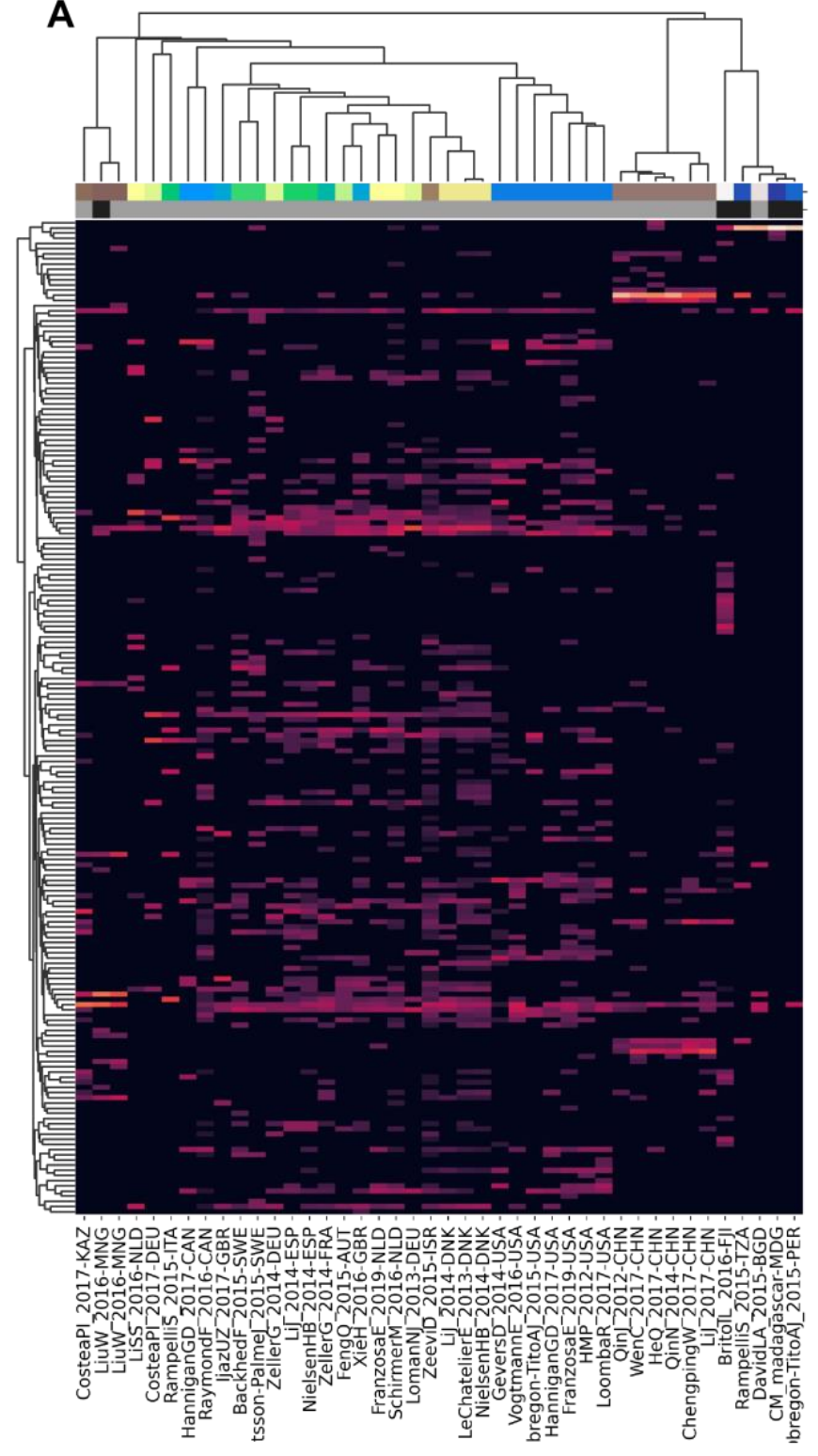

Country

$\begin{array}{lllll}\text { MDG } & \text { ITA } & \text { AUT } & \text { RUS } & \text { SGP } \\ \text { TZA } & \text { ESP } & \text { DEU } & \text { ISL } & \text { BRN } \\ \text { PER } & \text { SWE } & \text { NLD } & \text { ISR } & \text { IDN } \\ \text { USA } & \text { SVK } & \text { DNK } & \text { KAZ } & \text { MYS } \\ \text { CAN } & \text { HUN } & \text { NOR } & \text { MNG } & \text { BGD } \\ \text { GBR } & \text { EST } & \text { FIN } & \text { CHN } & \text { FJI } \\ \text { FRA } & & & & \end{array}$

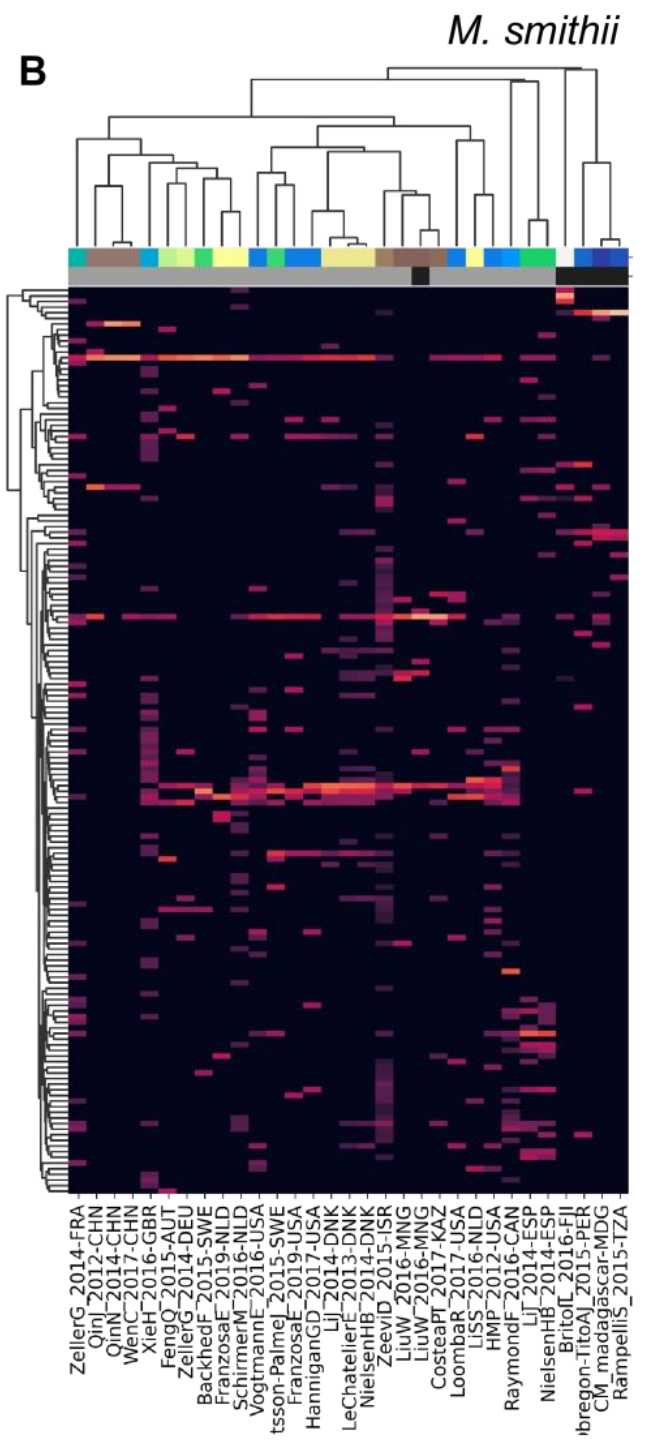


Figure 6: Patterns in strain dominance according to geography and lifestyle across thousands of publicly available metagenomes in dozens of independent studies for two common members of the human gut microbiome. Columns represent collections of samples from individual studies and are further segmented by country and lifestyle (westernized or not). Rows represent strains inferred by StrainFacts. Cell colors reflect the fraction of samples in that study segment with that strain as the most abundant member. Study segments are omitted if they include fewer than 10 samples. Row ordering and the associated dendrogram reflect strain genotype distances, while the dendrogram for columns is based on their cosine similarity. Colors above the heatmap reflect the country in which samples were collected as well as whether samples were collected from individuals with a westernized lifestyle. Both a study identifier and the ISO 3166-ISO country-code are included in the column labels.

These general trends hold across the other three species. In M. smithii, independent studies in the same country often share very similar strain dominance patterns (e.g. see clustering of studies performed in each of China, Mongolia, Denmark, and Spain in Fig. 6B). In E. coli (Supplementary Fig. S3A), while many strains appear to be distributed globally, independent studies from China still cluster together based on patterns in strain dominance. Notably, in CAG279 (Supplementary Fig. S3B), studies with individuals in westernized societies do not cluster separately from the five other studies, suggesting that host lifestyle is not highly correlated with specific strains of this species. The variety of dominance patterns across the four species described here suggest that different mechanisms may drive intraspecific biogeography depending on the biology and natural history of the species. As the coverage of diverse microbiomes grows, StrainFacts will enable future studies disentangling the contributions of lifestyle, dispersal limitation and other factors in the global distribution of strains.

\section{Linkage disequilibrium decay suggests variation in recombination rates across microbial species}

Studying the population genetics of host-associated microbes has the potential to elucidate processes of transmission, diversification, and selection with implications for human health and perhaps even our understanding of human origins $(9,37)$. To demonstrate the application of StrainFacts to the study of microbial evolution, we examined patterns in pairwise LD, here calculated as the squared Pearson correlation coefficient (r2). This statistic can inform understanding of recombination rates in microbial populations $(19,38)$. Genome-wide, LD, summarized as the 90th percentile $\mathrm{r} 2$ (LD90, 39), was substantially higher for $E$. coli (mean of 0.24 ) than $A$. rectalis (0.04), M. smithii $(0.11)$, or CAG-279 (0.04), perhaps suggesting greater population structure in the species and less panmictic recombination.

We estimated LD distance-decay curves for SNPs, using a single, high-quality reference genome for each species to obtain estimates of pairwise distance between SNP sites. For all four species, adjacent SNPs were much more tightly linked, with an LD ${ }_{90}$ of $>0.999$. LD was still dramatically above background at 50 bases of separation, and fell rapidly with increasing distance (Fig. 7). The speed of this decay was different between species, which we characterized with the $L D_{1 / 2,90}$ : the distance at which the $L D_{90}$ was less than $50 \%$ of the value for adjacent SNPs (39). M. smithii exhibited by far the slowest decay, with a $L D_{1 / 2,90}$ of 520 bases, followed by $E$. coli at 93 bases, CAG-279 at 66, and $A$. rectalis at just 28 bases. This variation in decay profiles may reflect major differences in recombination rates across populations.

To validate our findings, we ran identical analyses with dereplicated genotypes from genomes in the GT-Pro reference database. Estimates of both LD and the distance-decay relationship are very similar between inferred and reference strains, reinforcing the value of genotypes inferred from metagenomes for microbial population genetics. Interestingly, for three of the four species (E. coli, A. rectalis, and M. smithii), LD estimates from StrainFacts strains were significantly higher than from references ( $p<1 e-10$ for all three by Wilcoxon test), while CAG-279 exhibited a 
trend toward the reverse. It is not clear what might cause these quantitative discrepancies, but they could reflect differences in the set of strains in each dataset. Future studies expanding this analysis to additional species will identify patterns in recombination rates across broader microbial diversity.
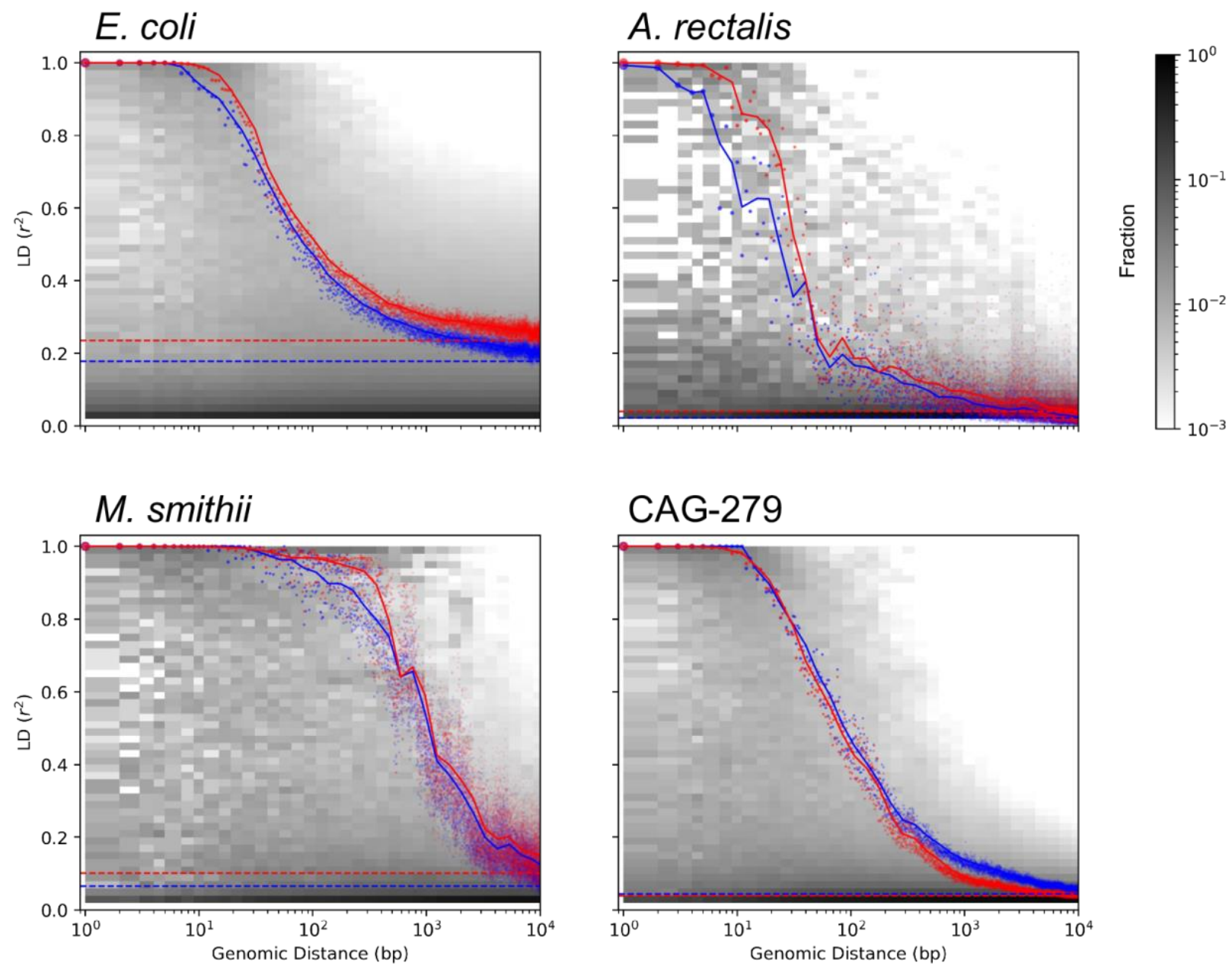

Figure 7: Pairwise $L D$ across genomic distance estimated from inferred genotypes for four species. $L D$ was calculated as $r 2$ and genomic distance between polymorphic loci is based on distances in a single, representative genome. The distribution of SNP pairs in each distance window is shown as a histogram with darker colors reflecting a larger fraction of the pairs in that $L D$ bin, and the $L D_{90}$ all for pairs at each distance is shown as points for inferred strains (red), along with an identical analysis on strains in the reference database (blue). LD9o in each histogram window (solid lines) and genome-wide (dashed lines) are also indicated.

\section{Discussion}

Here we have described StrainFacts, a novel tool for strain inference in metagenomic data. StrainFacts models metagenotype data using a fuzzy-genotype approximation, allowing us to estimate both the relative abundance of strains across samples as well as their genotypes. To accelerate analysis compared to the current state-of-the-art, we harness the differentiability of our model to apply modern, gradient-based optimization and GPU-parallelization. Consequently, StrainFacts can scale to tens-of-thousands of samples while inferring genotypes for hundreds of 
strains. On simulated benchmarks, we show that StrainFacts has comparable accuracy to Strain Finder, and we validate strain inferences in vivo against genotypes observed by single-cell genomics. Finally, we apply StrainFacts to a database of tens of thousands of metagenomes from the human microbiome to estimate strains de novo, allowing us to characterize strain diversity, biogeography, and population genetics, without the need for cultured isolates.

Beyond Strain Finder, other alternatives exist for strain inference in metagenomic data. While we do not directly compare to DESMAN, runtimes of several hours have been reported for that tool on substantially smaller simulated datasets (22), and hence we believe that StrainFacts is likely the fastest implementation of the metagenotype deconvolution approach. Still other methods apply regularized regression (e.g. Lasso 8) to decompose metagenotypes-essentially solving the abundance half of the deconvolution problem but not the genotypes half-or look for previously determined strain signatures (e.g. k-mers 40) based on known strain genotypes. However, both of these require an a priori database of the genotypes that might be present in a sample. An expanding database of strain references can make these approaches increasingly useful, and StrainFacts can help to build this reference.

Several studies avoid deconvolution by directly examining allele frequencies inferred from metagenotypes. While consensus $(11,41)$ or phasing (19) approaches can accurately recover genotypes in some cases, their use is limited to low complexity datasets, with sufficient sequencing depth and low strain heterogeneity. Likewise, measuring the dissimilarity of metagenotypes among pairwise samples indicates shared strains (42), but this approach risks confounding strain mixing with genotype similarity. Finally, assembly (43) and read-based methods (44) for disentangling strains are most applicable when multiple SNPs can be found in each sequencing read. With ongoing advancements in long-read (45) and read-cloud sequencing $(46,47)$, these approaches will become increasingly feasible. Thus, StrainFacts occupies the same analysis niche as Strain Finder and DESMAN, and it expands upon these reliable approaches by providing a scalable model fitting procedure.

Besides enabling more computationally efficient inference, fuzzy genotypes may also be more robust to deviations from model assumptions. For instance, an intermediate genotype could be a satisfactory approximation when gene duplications or deletions are present in some strains. While we do not explore the possibility here, fuzzy genotypes may also provide a heuristic for capturing uncertainty in strain genotypes. For example, future work could consider propagating intermediate genotype values instead of discretizing them.

StrainFacts builds on recent advances in metagenotyping, in particular our analyses harnessed GT-Pro (16) to greatly accelerate SNP counting in metagenomic reads. While we leave a comparison of StrainFacts performance on the outputs of other metagenotypers to future work, StrainFacts itself is agnostic to the source of input data. It would be straightforward to extend StrainFacts to operate on loci with more than two alleles, using metagenotypes from a tool other than GT-Pro. It would also be interesting to extend StrainFacts to use SNPs outside the core genome that vary in their presence across strains.

Combined with the explosive growth in publicly available metagenomic data and the development of rapid metagenotyping tools, StrainFacts enables the de novo exploration of intraspecific microbial diversity at a global scale and on well-powered cohorts with thousands of samples. Future applications could examine intraspecific associations with disease, track the history of recombination between microbial subpopulations, and measure the global transmission of host-associated microbes across human populations. 


\section{Endmatter}

\section{Funding Statement}

BJS was supported by National Institutes of Health grant number 5T32DK007007 and from the UCSF Initiative for Digital Transformation in Computational Biology \& Health. BJS, ZJS, and KSP were supported by National Science Foundation grant number 1563159 and by The Gladstone Institutes. BJS, XL, ZJS, AA, and KSP were supported by funding from Chan Zuckerberg Biohub.

\section{Acknowledgements}

Barbara Engelhardt provided valuable feedback on this project.

\section{Competing Interests}

KSP is on the scientific advisory board of Phylagen.

\section{Author Contributions}

- BJS: conceptualization, data curation, formal analysis, methodology, software, visualization, writing - original draft, writing - review \& editing

- $\quad \mathrm{XL}$ : investigation, data curation, methodology, writing - original draft, writing - review \& editing

- $\quad$ ZJS: data curation, methodology, writing - review \& editing

- $\quad A A$ : funding acquisition, supervision

- KSP: conceptualization, writing - review \& editing, funding acquisition, supervision

\section{Data Availability Statement}

Metagenomic and single-cell sequencing data from the FMT study will be uploaded to the SRA under BioProject PRJNA737472. Publicly available metagenomes are available under various other accessions described in (16). Strain genotypes from the GT-Pro reference database are publicly available at <https://fileshare.czbiohub.org/s/waXQzQ9PRZPwTdk>. All other code and metadata needed to reproduce these results are available at

https://github.com/bsmith89/StrainFacts-manuscript. 


\section{Supplementary Materials}

\section{Supplementary Results}

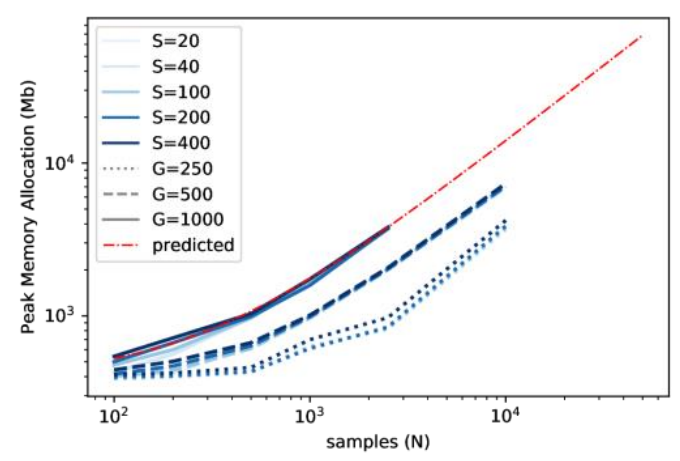

Figure S1: Maximum memory allocation across varying numbers of strains (S, line shade), SNPs (G, line style), and samples is plotted for StrainFacts models. Median of 9 replicate runs is shown. Maximum memory requirements are extrapolated to higher numbers of samples for a model with 1000 SNP sites (red line). An abridged version of this plot is included as Fig. 2.

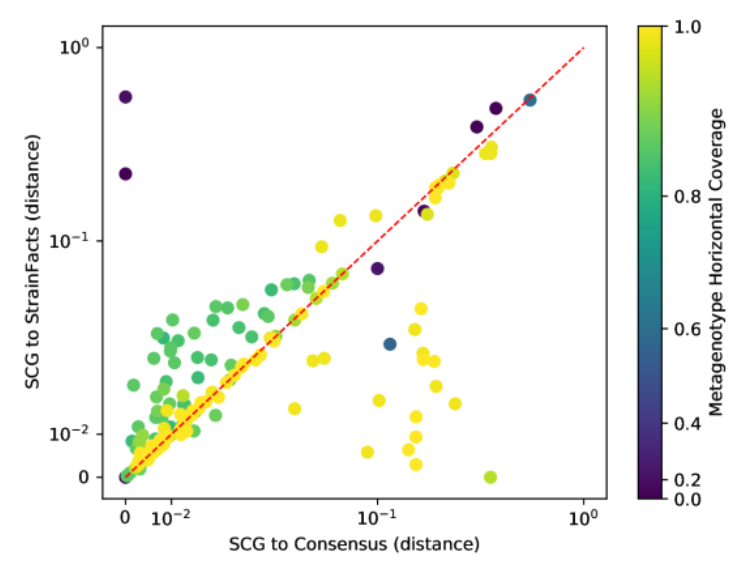

Figure S2: Inferred strains reflect genotypes from a single-cell sequencing experiment. Distance between observed SCGs and StrainFacts inferences ( $X$-axis) versus consensus genotypes ( $Y$-axis), plotted as in Fig. 4. Points below and to the right of the red dotted line reflecting an improvement of our method over the consensus, based on the normalized, best-match Hamming distance. Each dot represents an individual SCG reflecting a putative genotype found in the analysed sample. SCGs from all species found in one sample are represented, and marker colors reflect the metagenotype horizontal coverage for that species. Axes are on a "symmetric" log scale, with linear placement of values below 10-2. 
bioRxiv preprint doi: https://doi.org/10.1101/2022.02.01.478746: this version posted Februarv 4. 2022. The copvright holder for this preprint (which was not certified by peer review) is the author/funder, who has granted bioRxiv a license to display the preprint in perpetuity. It is made available under aCC-BY 4.0 International license.

Strain Dominance (frac. samples)

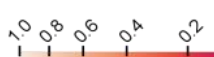

Westernized

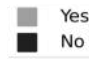

E. coli

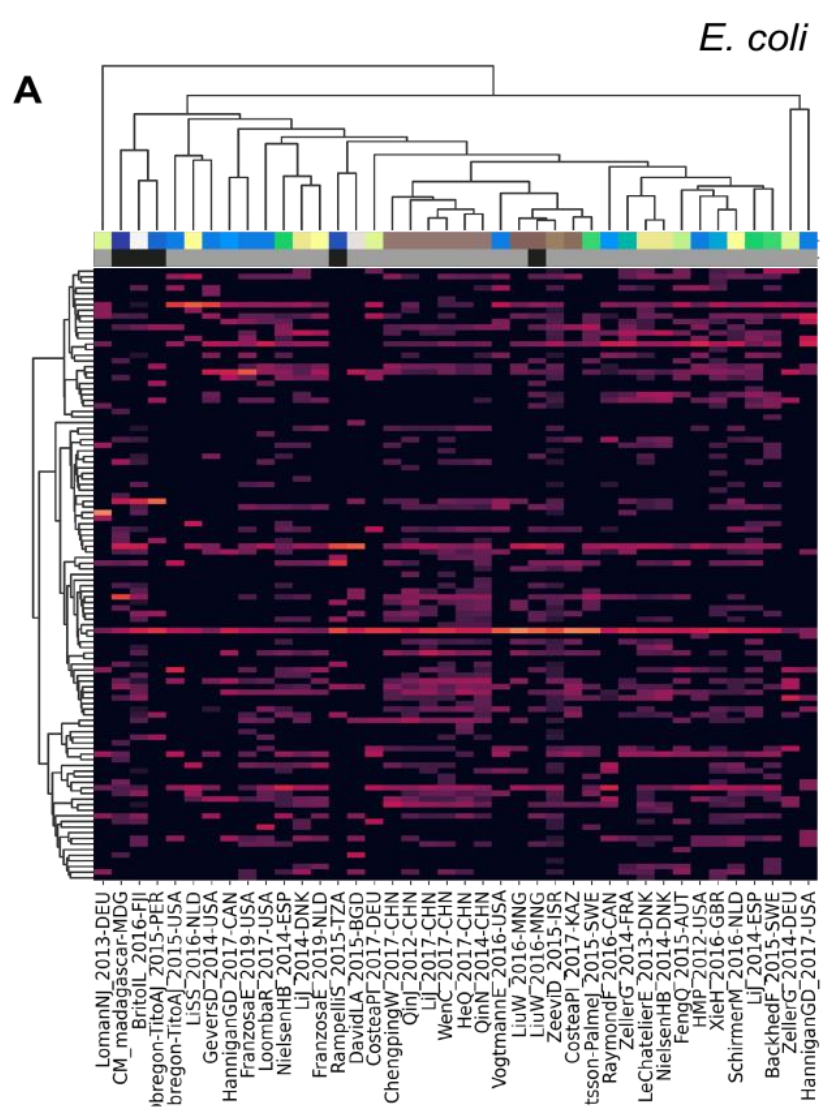

B

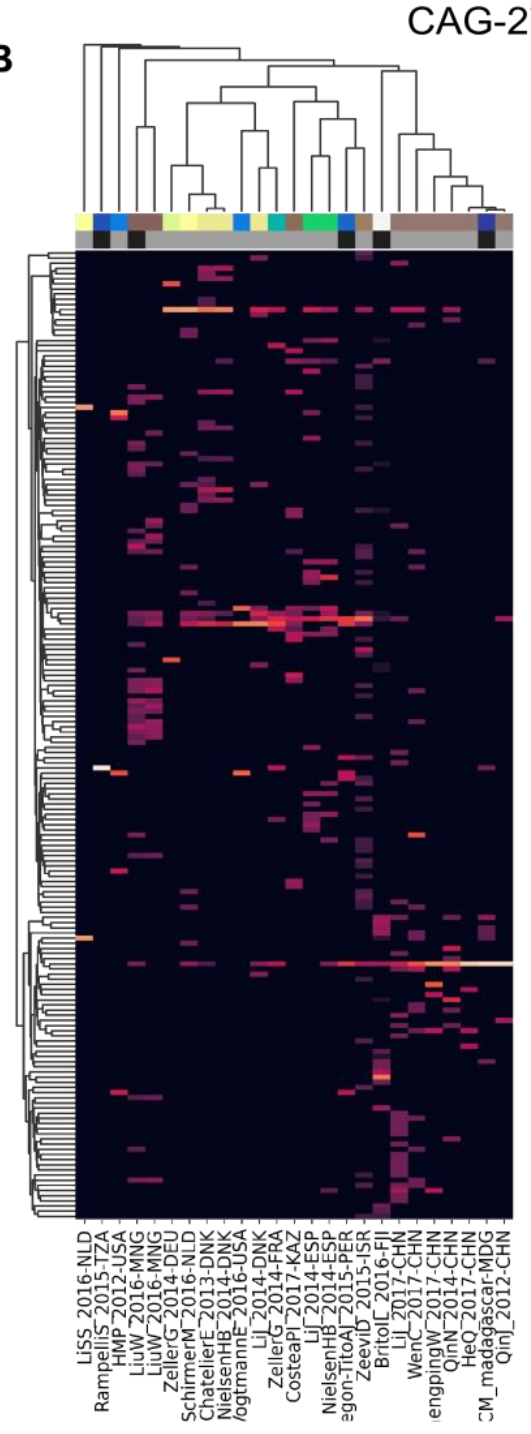

Figure S3: Patterns in strain dominance according to geography and lifestyle across thousands of publicly available metagenomes in dozens of independent studies for two additional members of the human gut microbiome. Visual elements are identical to Fig. 6: Columns represent collections of samples from individual studies and are further segmented by country and lifestyle (westernized or not). Rows represent strains inferred by StrainFacts. Cell colors reflect the fraction of samples in that study segment with that strain as the most abundant member. Study segments are omitted if they include fewer than 10 samples. Row ordering and the associated dendrogram reflect strain genotype distances, while the dendrogram for columns is based on their cosine similarity. Colors above the heatmap reflect the country in which samples were collected as well as whether samples were collected from individuals with a westernized lifestyle. Both a study identifier and the ISO 3166-ISO country-code are included in the column labels. 


\section{Supplementary Methods}

\section{The shifted, scaled Dirichlet distribution}

The k-dimensional SSD is a $2 \mathrm{k}+1$ parameter family which includes the Dirichlet distribution as a special case, and is defined by Aitchenson "perturbation" $(\oplus)$ and "powering" $(\odot)$ operations (48) applied to a Dirichlet-distributed random variable. Given a random variable $\mathbf{X} \sim$ $\operatorname{Dirichlet}(\boldsymbol{\alpha}), \boldsymbol{\alpha} \in \mathbb{R}^{K}, \mathbf{X} \in \mathcal{S}^{D}$ if $\mathbf{Y}=\mathbf{p} \oplus(a \odot \mathbf{X}), a \in \mathbb{R}_{+}, \mathbf{p} \in \mathcal{S}^{K}, \mathbf{Y} \in \mathcal{S}^{K}$ then $\mathbf{Y} \sim \operatorname{SSD}(\boldsymbol{\alpha}, \mathbf{p}, a)$.

In this work, we limit our use of this distribution to $\alpha=\mathbf{1}$, i.e. $\mathbf{X}$ distributed uniformly on the Ksimplex before powering and perturbation. For values of $a>1$, the probability mass shifts towards the edges of the simplex, and we use this property in order to induce sparsity in our estimates.

\section{Parameter initialization and optimization}

In select initial values of $\Pi$ and $\Gamma$ using an NMF based approach. First, we transform metagenotypes from counts to an $N \times G \times 2$ matrix of allele frequencies, and stack this into an $N \times 2 G$ matrix, $\mathbf{P}^{\prime}$, with columns of reference alleles followed by columns of alternative alleles. We then use canonical NMF-implemented in the scikit-learn library (49)_to factorize this data matrix into $\boldsymbol{\Pi}^{\prime}$ and $\boldsymbol{\Gamma}^{\prime}$, where $\mathbf{P}^{\prime} \approx \boldsymbol{\Pi}^{\prime} \times \boldsymbol{\Gamma}^{\prime}$ with a shared, inner dimension of size $S$. After reversing the stacking of alleles, we get back a matrix $\Gamma^{\prime \prime} \in \mathbb{R}_{+}^{S \times G \times 2}$. Since $\Pi^{\prime}$ and $\Gamma^{\prime \prime}$ likely do not conform to the constraints of strain deconvolution, we transform them into initial values as follows: $\boldsymbol{\Gamma}_{\text {init }}=$ $\mathcal{C}\left(1 / \vec{c} * \Gamma^{\prime \prime}\right)$ and $\boldsymbol{\Pi}_{\text {init }}=\mathcal{C}\left(\vec{c} * \boldsymbol{\Pi}^{\prime}\right)$ where $c_{s}=\frac{1}{G} \sum_{g}\left(\gamma_{s g 0}+\gamma_{s g 1}\right), *$ denotes element-wise multiplication, and $\mathcal{C}(\cdot)$ is normalization over the last dimension to the standard simplex (i.e. summing-to-one).

Model parameters are transformed to the unconstrained space using Pyro's built-in defaults. Parameters other than $\Pi$ and $\boldsymbol{\Gamma}$ are initialized randomly to a point on the interval $(-2,2)$ in the transformed space. We then apply the Adamax algorithm for stochastic gradient descent using an initial learning rate $1 r$ init. To increase the probability of finding a global maximum, we take a prior annealing approach (50): for an initial n_wait number of steps of the optimization routine, the hyperparameters $\gamma^{*}$ and $\rho^{*}$ are set to initial values with less stringent regularization and are then exponentially relaxed to their final values during the next $n$ _anneal steps. After this annealing period, we continue taking gradient steps until the value of the loss function has not improved for 100 steps, at which point we halve the learning rate. Optimization is stopped when the learning rate falls below a minimum threshold, Ir_min.

\section{Fitting full length genotypes}

Because many metagenotypes had more than 5,000 SNP sites, we use a refitting approach to get full length strain genotypes. This is accomplished by conditioning our model on both the observed data and the previously estimated $\Pi$. In addition, we update the value of two of the hyperparameters; we set $\gamma^{*}=1.0$, and $\alpha^{*}=200$. After refitting the other parameters, this results in a new estimate of $\boldsymbol{\Gamma}$. Since SNPs are statistically separable when $\boldsymbol{\Pi}$ is conditioned out, this allows us to iteratively refit arbitrary subsets of SNPs before recombining them into a full length genotype matrix. 


\section{Single-cell genomic sequencing}

\section{Cell isolation from stool samples}

Bacterial cells were isolated from fecal samples according to previously published protocol (51) with modifications. Briefly, 0.2-0.5 g of fecal samples were homogenized in $10 \mathrm{~mL}$ of PBS buffer by vertexing. After filtering with a $50 \mu \mathrm{m}$ cell strainer (Corning, 431752) to remove most of the fecal particles, the flow through suspension was loaded on top of $3.5 \mathrm{~mL}$ of $80 \%$ Nycodenz $®$ (Cosmo Bio USA, AXS-1002424) in a $15 \mathrm{~mL}$ conical tube. The tube was centrifuged for $40 \mathrm{~min}$ at $4{ }^{\circ} \mathrm{C}(4700 \times \mathrm{g})$. The layer corresponding to microbiota was extracted and washed with PBS for 3 times. The cells were directly processed for hydrogel encapsulation or stored in DNA/RNA shield (Zymo Research, R1100-50) at $-80^{\circ} \mathrm{C}$ for long term storage.

\section{High-throughput single bacterial sequencing}

Barcoded single cell bacterial sequencing libraries were constructed by modified SiC- protocol Seq leveraging Mission Bio Tapestri (52).

\section{Cell encapsulation in hydrogel beads}

Cell suspension (100 million per $\mathrm{mL}$ in PBS, $500 \mu \mathrm{L}$ ) was mixed with $500 \mu \mathrm{L}$ of polyacrylamide precursor solution with $12 \%$ acrylamide(Thermo Scientific, AAJ62480AP), $1 \%$ N,N'bis(acryloyl)cystamine (Sigma, A5912), $20 \mathrm{mM}$ Tris (pH 8.0), 0.6\% sodium persulfate (Sigma, 216232), and $20 \mathrm{mM} \mathrm{NaCl}$. After adding $1 \mathrm{~mL}$ of HFE 7500 with $2 \%$ surfactant (008FluoroSurfactant, RanBiotechnologies), heterogenous droplets were generated by passing the oil/aqueous mixture through a syringe with $23.5 \mathrm{G}$ blunt needle for 5 time. $20 \mu \mathrm{L}$ of N,N,N',N'tetramethylethylenediamine (Sigma, 411019) was added into the emulsion and the emulsion was incubated at $70{ }^{\circ} \mathrm{C}$ for $30 \mathrm{~min}$ and at room temperature for 1 hour for gelation. The emulsion was centrifuged at 1000xG for $10 \mathrm{~min}$ and the oil layer was removed by a gel loading tip. To the hydrogel layer, $1 \mathrm{~mL}$ of $20 \%$ PFO $(1 \mathrm{H}, 1 \mathrm{H}, 2 \mathrm{H}, 2 \mathrm{H}$-perfluoro-1-octanol, Sigma, 370533) in HFE 7500 and the mixture was vortexed and shaking for 10 min to break the emulsions. After centrifugation at $1000 \times \mathrm{x}$ for $10 \mathrm{~min}$, PFO were removed, and the hydrogel beads were then washed with PBS with $0.4 \%$ tween 20 for 3 times. The beads were then resuspended in $40 \%$ sucrose in PBS with $0.4 \%$ tween 20 . A differential velocity centrifugation was performed to select the hydrogel beads within the size between 5 to $25 \mu \mathrm{m}$.

\section{Cell lysis and DNA purification in hydrogel beads}

$100 \mu \mathrm{L}$ of beads were treated in a solution of $1 \mathrm{~mL}$ TE buffer solution containing $2.5 \mathrm{mM}$ EDTA (Teknova), 10mM NaCl (Sigma-Aldrich), 2U zymolyase, $5 \mathrm{U}$ Lysostaphin, $50 \mathrm{U}$ mutanolysin, and $20 \mathrm{mg} \mathrm{Lysozyme}$ at $37^{\circ} \mathrm{C}$ overnight. The lysate mixture was then centrifuged at $3000 \times \mathrm{G}$ for $3 \mathrm{~min}$, the supernatant removed, and $1 \mathrm{~mL}$ of TE buffer with $4 \mathrm{U}$ of Proteinase $\mathrm{K}, 1 \%$ triton $\mathrm{X} 100$ and $100 \mathrm{mM}$ of $\mathrm{NaCl}$ was added to digest cellular proteins. The solution is incubated at 50 ${ }^{\circ} \mathrm{C}$ for $30 \mathrm{~min}$. Following lysis, the beads was thoroughly washed to ensure complete removal of detergents and other chemicals which may inhibit the downstream reactions. The washes were performed in $10 \mathrm{~mL}$ volume with centrifugation magnitudes of $3000 \mathrm{xg}$ between washes.

\section{Tagmentsation reagents}

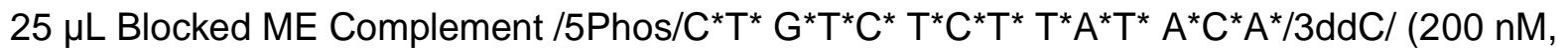
IDT), $25 \mu \mathrm{L}$ Tn5-Fwd-oligo GTACTCGCAGTAGTCAGATGTGTATAAGAGACAG (100 nM, IDT), and $25 \mu \mathrm{L}$ Tn5-Rev-oligo TACCCTTCCAATTTAACCCTCCAAGATGTGTATAAGAGACAG (100 $\mathrm{nM}$, IDT) and $25 \mu \mathrm{L}$ Tris buffer were mixed well in a PCR tube by pipetting. The mixture was 
incubated on a thermal cycler using the following program: $85^{\circ} \mathrm{C}$ for $2 \mathrm{~min}$, cools to $20^{\circ} \mathrm{C}$ with a ramping rate at $0.1^{\circ} \mathrm{C} / \mathrm{s}, 20^{\circ} \mathrm{C}$ for $1 \mathrm{~min}$, then hold at $4^{\circ} \mathrm{C}$ with lid at $105^{\circ} \mathrm{C} .100 \mu \mathrm{L}$ of glycerol was then added into the annealed oligo. The unloaded Tn5 $(1 \mathrm{mg} / \mathrm{mL}$, expressed by QB3 MacroLab, Berkeley, CA.) was diluted at a 1:1 ratio in the Illumina dilution buffer ( $50 \%$ Glycerol, $100 \mathrm{mM} \mathrm{NaCl}, 0.1 \mathrm{mM}$ EDTA, $1 \mathrm{mM} \mathrm{DTT}$, and 0.1\% NP40 in $50 \mathrm{mM}$ Tris-HCl pH 7.5 buffer), followed by mixing at a 1:1 ratio with the pre-annealed adapter/glycerol mix. The mixture was incubated at room temperature for 30 min then stored at $-20^{\circ} \mathrm{C}$.

\section{Single cell DNA tagmentaion}

The beads were resuspended in the density matching buffer $(10 \mathrm{mM} \mathrm{MgCl}, 1 \% \mathrm{NP} 40$, and $17 \%$ Optiprep in $20 \mathrm{mM}$ TAPS pH 7.0 buffer) to the final cell density of 3000 cells $/ \mu \mathrm{L} .25 \mu \mathrm{L}$ of assembled Tn5 was mixed with $25 \mu \mathrm{L}$ of tagmention buffer $(10 \mathrm{mM} \mathrm{MgCl}, 10 \mathrm{mM}$ DTT in 20 $\mathrm{mM}$ TAPS pH 7.0 buffer). The MissionBio Tapestri DNA cartridge and a $0.2 \mathrm{~mL}$ PCR tube were mounted onto the Tapestri instrument. $50 \mu \mathrm{L}$ of the beads in density matching buffer was loaded into the reservoir 2 (the reservoir for cell suspension), $50 \mu \mathrm{L}$ of the Tn5 in tagmentation buffer was loaded into reservoir 1 (the reservoir for lysis buffer), and $200 \mu \mathrm{L}$ of Encapsulation oil was loaded into reservoir 3. After applying the DNA gasket on top of the cartridge and closing the instrument lid, droplets were generated by running the Step1: Encapsulation program. The droplets were incubated at $37^{\circ} \mathrm{C}$ for $60 \mathrm{~min}$ and then $50^{\circ} \mathrm{C}$ for $30 \mathrm{~min}$.

\section{Barcoding PCR}

Barcoding droplet PCR were performed according to the MissionBio Tapestri protocol with minor modification. 8 PCR tubes and DNA cartridge were mounted onto the Tapestri instrument. $200 \mu \mathrm{L}$ and $500 \mu \mathrm{L}$ of Electrode solution were loaded into reservoirs 4 and 5 respectively. After running the Priming program, $5 \mu \mathrm{L}$ of reverse barcoding oligo was mixed with $295 \mu \mathrm{L}$ MissionBio Barcoding Mix and loaded into reservoir 8 of the DNA cartridge. The droplets from previous step ( $~ 80 \mathrm{uL}), 200 \mu \mathrm{L}$ of barcoding beads, and $1.25 \mathrm{~mL}$ of Barcoding oil were loaded into reservoir 6 , 7 and 9, respectively. After applying the DNA gasket on top of the cartridge and closing the lid, the droplets were merged with barcoding beads and PCR reagents by running the Cell Barcoding program on the Tapestri instrument. The droplets collected in the 8 PCR tubes were treated with UV for 8 min (Analytik Jena Blak-Ray XX-15L UV light source) and the bottom layer of oil in each tube were removed using a gel loading tip to leave up to $100 \mu \mathrm{L}$ of droplets. The tubes were then thermo-cycled on a PCR instrument with the following program: 10 min at $72^{\circ} \mathrm{C}$ for 1 cycle, $3 \mathrm{~min}$ at $95^{\circ} \mathrm{C}$ for $1 \mathrm{cycle},\left(15 \mathrm{~s}\right.$ at $95^{\circ} \mathrm{C}, 15 \mathrm{~s}$ for $55^{\circ} \mathrm{C}$, and 2 min at $\left.72^{\circ} \mathrm{C}\right)$ for 20 cycles, and $5 \mathrm{~min}$ at $72^{\circ} \mathrm{C}$ for 1 cycle.

\section{Sequencing library preparation}

The thermal cycled droplets in the 8 PCR tubes were carefully transferred into two $1.5 \mathrm{~mL}$ centrifuge tubes (4 PCR tube content in each). If there were merged droplets present, they were carefully removed using a $2 \mu \mathrm{L}$ pipette. $20 \mu \mathrm{L}$ PFO were added into each tube and mixed well by vortex. After centrifuging the top aqueous layers in each tube was transferred into new 1.5 $\mathrm{mL}$ tubes and water was added to bring the total volume to $400 \mu \mathrm{L}$. The barcoding product was purified using 0.7X Ampure XP beads (Beckman Coulter, A63882) and eluted into $60 \mu \mathrm{L} \mathrm{H} 2 \mathrm{O}$ and stored at $-20^{\circ} \mathrm{C}$ until next step. The concentrations of the barcoding product were measured with Qubit ${ }^{\mathrm{TM}}$ 1X dsDNA Assay Kits (ThermoFisher, Q33230).

The sequencing library were then prepared by attaching P5 and P7 sequences to the barcoding products using Nextera primers. The library PCR reactions were performed with 25 uL Kapa HiFi Master mix 2X, 5 uL Library P5 index primer (4 uM), 5 uL Library P7 index primer (4 uM), $10 \mathrm{uL}$ purified barcoding products (normalized to $0.2 \mathrm{ng} / \mathrm{uL}$ ), and $5 \mathrm{uL}$ of nuclease free water. 
The PCR tubes were thermal cycled with the following program: $3 \mathrm{~min}$ at $95^{\circ} \mathrm{C}$ for $1 \mathrm{cycle},(20 \mathrm{~s}$ at $98^{\circ} \mathrm{C}, 20 \mathrm{~s}$ for $62^{\circ} \mathrm{C}$, and $45 \mathrm{~s}$ at $72^{\circ} \mathrm{C}$ ) for 10 cycles, and 2 min at $72^{\circ} \mathrm{C}$ for 1 cycle. The sequencing library was purified with 0.69 X Ampure XP beads and eluted into 12 uL nucleasefree water. The library was quantified with Qubit ${ }^{\mathrm{TM}}$ 1X dsDNA Assay Kits and DNA HS chips on bioanalyzer or D5000 ScreenTape (Agilent, 5067- 5588) on Tapestation (Agilent, G2964AA). The libraries were pooled and paired-end sequenced by Novogene with a partial lane on Illumina NovaSeq 6000.

\section{Single cell read files preparation}

Sequencing data were processed using a custom python script (mb_barcode_and_trim.py) available on GitHub at https://github.com/AbateLab/MissonBioTools. For all reads, combinatorial cell barcodes were parsed from Read 1, using cutadapt (v2.4) and matched to a barcode whitelist. Barcode sequences within a Hamming distance of 1 from a whitelist barcode were corrected. Reads with valid barcodes were trimmed with cutadapt to remove $5^{\prime}$ and $3^{\prime}$ adapter sequences and demultiplexed into single-cell FASTQ files by barcode sequences using the script demuxbyname.sh from the BBMap package (v.38.57).

\section{References}

1. Loman NJ, Constantinidou C, Christner M, Rohde H, Chan JZ-M, Quick J, Weir JC, Quince C, Smith GP, Betley JR, et al. A culture-independent sequence-based metagenomics approach to the investigation of an outbreak of Shiga-toxigenic Escherichia coli O104:H4. JAMA (2013) 309:1502-1510. doi: 10.1001/jama.2013.3231

2. Shoemaker NB, Vlamakis H, Hayes K, Salyers AA. Evidence for Extensive Resistance Gene Transfer amongBacteroides spp. And among Bacteroides and Other Genera in the Human Colon. Appl Environ Microbiol (2001) doi: 10.1128/AEM.67.2.561-568.2001

3. Haiser HJ, Seim KL, Balskus EP, Turnbaugh PJ. Mechanistic insight into digoxin inactivation by Eggerthella lenta augments our understanding of its pharmacokinetics. Gut Microbes (2014) 5:233-238. doi: 10.4161/gmic.27915

4. Patrick S, Blakely GW, Houston S, Moore J, Abratt VR, Bertalan M, Cerdeño-Tárraga AM, Quail MA, Corton N, Corton C, et al. Twenty-eight divergent polysaccharide loci specifying within- and amongst-strain capsule diversity in three strains of Bacteroides fragilis. Microbiology 156:3255-3269. doi: 10.1099/mic.0.042978-0

5. Martens E, Ostrowski M, Rosa SL, Kunath B, Robertson A, Pereira G, Hagan L, Varghese N, Qiu L, Yao T, et al. The Food Additive Xanthan Gum Drives Adaptation of the Human Gut Microbiota. [preprint]. In Review (2021). doi: 10.21203/rs.3.rs-607163/v1

6. Case RJ, Boucher Y, Dahllöf I, Holmström C, Doolittle WF, Kjelleberg S. Use of 16S rRNA and rpoB Genes as Molecular Markers for Microbial Ecology Studies. Appl Environ Microbiol (2007-January) doi: 10.1128/AEM.01177-06

7. Nayfach S, Roux S, Seshadri R, Udwary D, Varghese N, Schulz F, Wu D, Paez-Espino D, Chen I-M, Huntemann M, et al. A genomic catalog of Earth's microbiomes. Nat Biotechnol (2020)1-11. doi: 10.1038/s41587-020-0718-6

8. Albanese D, Donati C. Strain profiling and epidemiology of bacterial species from metagenomic sequencing. Nat Commun (2017) 8:2260. doi: 10.1038/s41467-017-02209-5 
9. Garud NR, Pollard KS. Population Genetics in the Human Microbiome. Trends in Genetics (2019) 0: doi: 10.1016/j.tig.2019.10.010

10. Yan Y, Nguyen LH, Franzosa EA, Huttenhower C. Strain-level epidemiology of microbial communities and the human microbiome. Genome Med (2020) 12:71. doi: 10.1186/s13073-02000765-y

11. Truong DT, Tett A, Pasolli E, Huttenhower C, Segata N. Microbial strain-level population structure and genetic diversity from metagenomes. Genome Res (2017) 27:626-638. doi: $10.1101 /$ gr.216242.116

12. Beghini F, Mclver LJ, Blanco-Míguez A, Dubois L, Asnicar F, Maharjan S, Mailyan A, Manghi $P$, Scholz M, Thomas AM, et al. Integrating taxonomic, functional, and strain-level profiling of diverse microbial communities with bioBakery 3. eLife (2021) 10:e65088. doi: 10.7554/eLife.65088

13. Costea PI, Hildebrand F, Arumugam M, Bäckhed F, Blaser MJ, Bushman FD, Vos WM de, Ehrlich SD, Fraser CM, Hattori M, et al. Enterotypes in the landscape of gut microbial community composition. Nat Microbiol (2017) Accepted: doi: 10.1038/s41564-017-0072-8

14. Olm MR, Crits-Christoph A, Bouma-Gregson K, Firek BA, Morowitz MJ, Banfield JF. inStrain profiles population microdiversity from metagenomic data and sensitively detects shared microbial strains. Nat Biotechnol (2021)1-10. doi: 10.1038/s41587-020-00797-0

15. Nayfach S, Rodriguez-Mueller B, Garud N, Pollard KS. An integrated metagenomics pipeline for strain profiling reveals novel patterns of bacterial transmission and biogeography. Genome Res (2016) 26:1612-1625. doi: 10.1101/gr.201863.115

16. Shi ZJ, Dimitrov B, Zhao C, Nayfach S, Pollard KS. Fast and accurate metagenotyping of the human gut microbiome with GT-Pro. Nat Biotechnol (2021)1-10. doi: 10.1038/s41587021-01102-3

17. Li SS, Zhu A, Benes V, Costea PI, Hercog R, Hildebrand F, Huerta-Cepas J, Nieuwdorp M, Salojärvi J, Voigt AY, et al. Durable coexistence of donor and recipient strains after fecal microbiota transplantation. Science (2016) 352:586-589. doi: 10.1126/science.aad8852

18. Costea PI, Coelho LP, Sunagawa S, Munch R, Huerta-Cepas J, Forslund K, Hildebrand F, Kushugulova A, Zeller G, Bork P. Subspecies in the global human gut microbiome. Mol Syst Biol (2017) 13:960. doi: 10.15252/msb.20177589

19. Garud NR, Good BH, Hallatschek O, Pollard KS. Evolutionary dynamics of bacteria in the gut microbiome within and across hosts. PLOS Biology (2019) 17:e3000102. doi: 10.1371/journal.pbio.3000102

20. O'Brien JD, Didelot X, Iqbal Z, Amenga-Etego L, Ahiska B, Falush D. A Bayesian Approach to Inferring the Phylogenetic Structure of Communities from Metagenomic Data. Genetics (2014) 197:925-937. doi: 10.1534/genetics.114.161299

21. Smillie CS, Sauk J, Gevers D, Friedman J, Sung J, Youngster I, Hohmann EL, Staley C, Khoruts A, Sadowsky MJ, et al. Strain Tracking Reveals the Determinants of Bacterial Engraftment in the Human Gut Following Fecal Microbiota Transplantation. Cell Host Microbe (2018) 23:229-240.e5. doi: 10.1016/j.chom.2018.01.003 
22. Quince C, Delmont TO, Raguideau S, Alneberg J, Darling AE, Collins G, Eren AM. DESMAN: A new tool for de novo extraction of strains from metagenomes. Genome Biol (2017) 18:1-22. doi: 10.1186/s13059-017-1309-9

23. Luo C, Knight R, Siljander H, Knip M, Xavier RJ, Gevers D. ConStrains identifies microbial strains in metagenomic datasets. Nat Biotechnol (2015) 33:1045-1052. doi: $10.1038 /$ nbt.3319

24. Chu ND, Crothers JW, Nguyen LTT, Kearney SM, Smith MB, Kassam Z, Collins C, Xavier R, Moses PL, Alm EJ. Dynamic Colonization of Microbes and Their Functions after Fecal Microbiota Transplantation for Inflammatory Bowel Disease. mBio (2021) doi:

10.1128/mBio.00975-21

25. Watson AR, Füssel J, Veseli I, DeLongchamp JZ, Silva M, Trigodet F, Lolans K, Shaiber A, Fogarty E, Quince C, et al. Adaptive ecological processes and metabolic independence drive microbial colonization and resilience in the human gut. bioRxiv (2021)2021.03.02.433653. doi: 10.1101/2021.03.02.433653

26. Smith BJ, Piceno Y, Zydek M, Zhang B, Syriani LA, Terdiman JP, Kassam Z, Ma A, Lynch SV, Pollard KS, et al. Strain-resolved analysis in a randomized trial of antibiotic pretreatment and maintenance dose delivery mode with fecal microbiota transplant for ulcerative colitis. (2022) doi: 10.1101/2021.08.07.21261556

27. Schmidt MN, Winther O, Hansen LK. Bayesian Non-negative Matrix Factorization. In: Adali T, Jutten C, Romano JMT, Barros AK, editors. Independent Component Analysis and Signal Separation. Lecture Notes in Computer Science. Berlin, Heidelberg: Springer (2009). p. 540-547 doi: 10.1007/978-3-642-00599-2_68

28. Monti GS, Mateu-Figueras G, Pawlowsky-Glahn V, Egozcue JJ. The shifted-scaled Dirichlet distribution in the simplex. (2011)

29. Bingham E, Chen JP, Jankowiak M, Obermeyer F, Pradhan N, Karaletsos T, Singh R, Szerlip P, Horsfall P, Goodman ND. Pyro: Deep Universal Probabilistic Programming. J Mach Learn Res (2019) 20:1-6. http://jmlr.org/papers/v20/18-403.html [Accessed April 8, 2021]

30. Paszke A, Gross S, Massa F, Lerer A, Bradbury J, Chanan G, Killeen T, Lin Z, Gimelshein N, Antiga L, et al. PyTorch: An Imperative Style, High-Performance Deep Learning Library. Advances in Neural Information Processing Systems. ancouver, Canada: Curran Associates, Inc. (2019)

https://proceedings.neurips.cc/paper/2019/hash/bdbca288fee7f92f2bfa9f7012727740 Abstract.html [Accessed January 30, 2022]

31. Almeida A, Nayfach S, Boland M, Strozzi F, Beracochea M, Shi ZJ, Pollard KS, Sakharova E, Parks DH, Hugenholtz P, et al. A unified catalog of 204,938 reference genomes from the human gut microbiome. Nat Biotechnol (2021) 39:105-114. doi: 10.1038/s41587-0200603-3

32. Kurtzer GM, Sochat V, Bauer MW. Singularity: Scientific containers for mobility of compute. PLOS ONE (2017) 12:e0177459. doi: 10.1371/journal.pone.0177459

33. Lozupone CA, Hamady M, Kelley ST, Knight R. Quantitative and Qualitative $\beta$ Diversity Measures Lead to Different Insights into Factors That Structure Microbial Communities. Appl Environ Microbiol (2007) 73:1576-1585. doi: 10.1128/AEM.01996-06 
34. Scanlan PD, Shanahan F, Marchesi JR. Human methanogen diversity and incidence in healthy and diseased colonic groups using mcrA gene analysis. BMC Microbiol (2008) 8:79. doi: 10.1186/1471-2180-8-79

35. Lagkouvardos I, Lesker TR, Hitch TCA, Gálvez EJC, Smit N, Neuhaus K, Wang J, Baines JF, Abt B, Stecher B, et al. Sequence and cultivation study of Muribaculaceae reveals novel species, host preference, and functional potential of this yet undescribed family. Microbiome (2019) 7:28. doi: 10.1186/s40168-019-0637-2

36. Scholz M, Ward DV, Pasolli E, Tolio T, Zolfo M, Asnicar F, Truong DT, Tett A, Morrow AL, Segata N. Strain-level microbial epidemiology and population genomics from shotgun metagenomics. Nat Methods (2016) 13:435-438. doi: 10.1038/nmeth.3802

37. Linz B, Balloux F, Moodley Y, Manica A, Liu H, Roumagnac P, Falush D, Stamer C, Prugnolle F, van der Merwe SW, et al. An African origin for the intimate association between humans and Helicobacter pylori. Nature (2007) 445:915-918. doi: 10.1038/nature05562

38. Vos M. Why do bacteria engage in homologous recombination? Trends Microbiol (2009) 17:226-32. doi: 10.1016/j.tim.2009.03.001

39. Vos PG, Paulo MJ, Voorrips RE, Visser RGF, van Eck HJ, van Eeuwijk FA. Evaluation of LD decay and various LD-decay estimators in simulated and SNP-array data of tetraploid potato. Theor App/ Genet (2017) 130:123-135. doi: 10.1007/s00122-016-2798-8

40. Panyukov VV, Kiselev SS, Ozoline ON. Unique k-mers as Strain-Specific Barcodes for Phylogenetic Analysis and Natural Microbiome Profiling. IJMS (2020) 21:944. doi: 10.3390/ijms21030944

41. Zolfo M, Tett A, Jousson O, Donati C, Segata N. MetaMLST: Multi-locus strain-level bacterial typing from metagenomic samples. Nucleic Acids Research (2017) 45:e7. doi: 10.1093/nar/gkw837

42. Podlesny D, Fricke WF. Microbial Strain Engraftment, Persistence and Replacement after Fecal Microbiota Transplantation. medRxiv (2020)2020.09.29.20203638. doi: 10.1101/2020.09.29.20203638

43. Li X, Saadat S, Hu H, Li X. BHap: A novel approach for bacterial haplotype reconstruction. Bioinformatics (2019) 35:4624-4631. doi: 10.1093/bioinformatics/btz280

44. Cleary B, Brito IL, Huang K, Gevers D, Shea T, Young S, Alm EJ. Detection of lowabundance bacterial strains in metagenomic datasets by eigengenome partitioning. Nat Biotechnol (2015) 33:1053-1060. doi: 10.1038/nbt.3329

45. Vicedomini R, Quince C, Darling AE, Chikhi R. Strainberry: Automated strain separation in low-complexity metagenomes using long reads. Nat Commun (2021) 12:4485. doi: 10.1038/s41467-021-24515-9

46. Kuleshov V, Snyder MP, Batzoglou S. Genome assembly from synthetic long read clouds. Bioinformatics (2016) 32:i216-i224. doi: 10.1093/bioinformatics/btw267

47. Kang JB, Siranosian B, Moss E, Andermann T, Bhatt A. Read cloud sequencing elucidates microbiome dynamics in a hematopoietic cell transplant patient. 2018 IEEE Int Conf Bioinforma Biomed BIBM (2018) doi: 10.1109/BIBM.2018.8621297 
48. Aitchison J. The statistical analysis of compositional data. London; New York: Chapman and Hall (1986).

49. Pedregosa F, Varoquaux G, Gramfort A, Michel V, Thirion B, Grisel O, Blondel M, Prettenhofer P, Weiss R, Dubourg V, et al. Scikit-learn: Machine learning in Python. J Mach Learn Res (2011) 12:2825-2830. http://www.jmlr.org/papers/v12/pedregosa11a.html

50. Neuwald AF, Liu JS. Gapped alignment of protein sequence motifs through Monte Carlo optimization of a hidden Markov model. BMC Bioinformatics (2004) 5:157. doi: 10.1186/14712105-5-157

51. Hevia A, Delgado S, Margolles A, Sánchez B. Application of density gradient for the isolation of the fecal microbial stool component and the potential use thereof. Sci Rep (2015) 5:16807. doi: 10.1038/srep16807

52. Lan F, Demaree B, Ahmed N, Abate A. SiC-Seq: Single-cell genome sequencing at ultra high-throughput with microfluidic droplet barcoding. Nat Biotechnol (2017) 35:640. doi: 


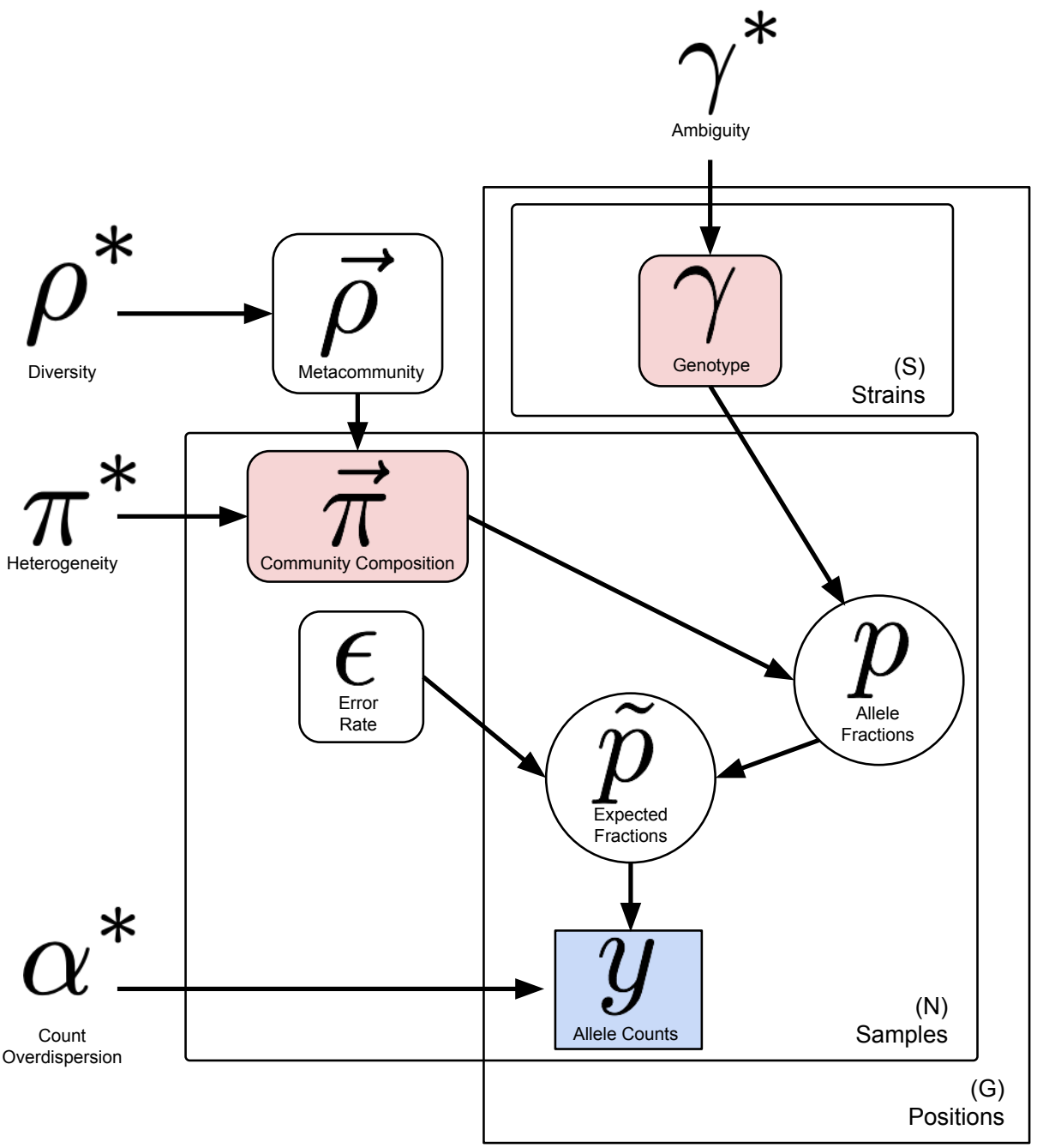




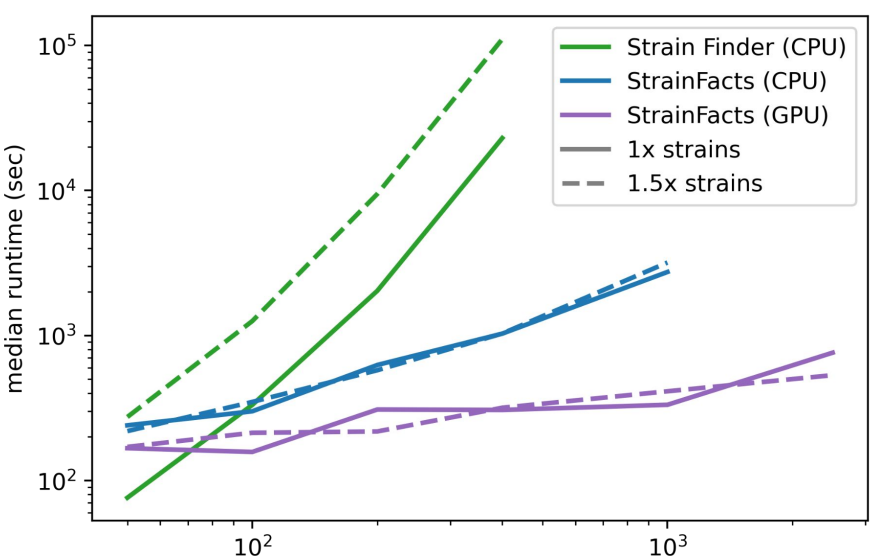

samples (N)

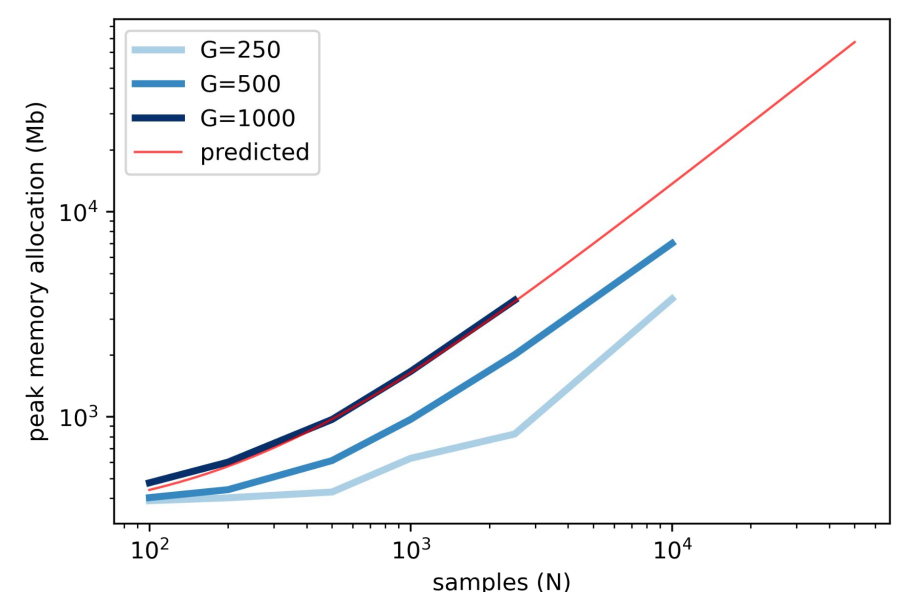



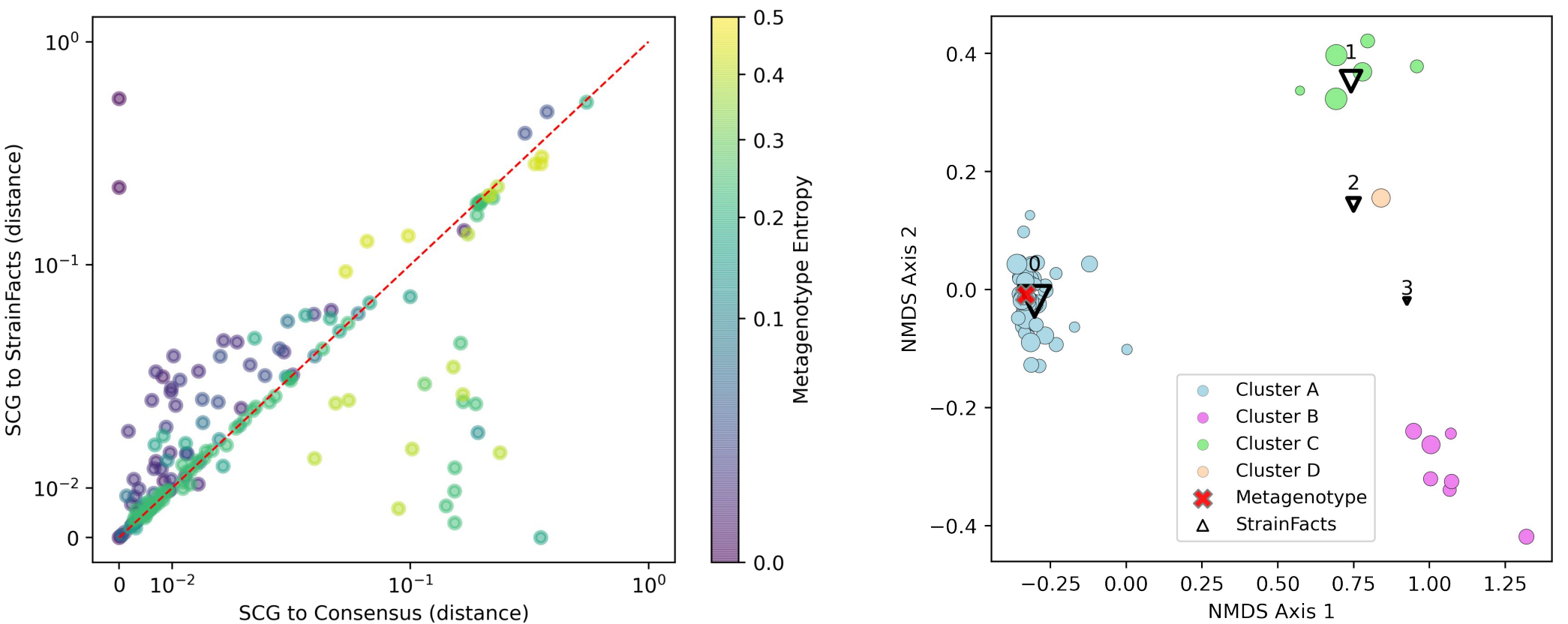
Strain Dominance (frac. samples)

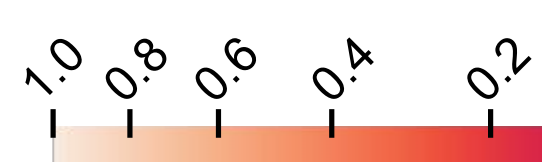

A. rectalis

A

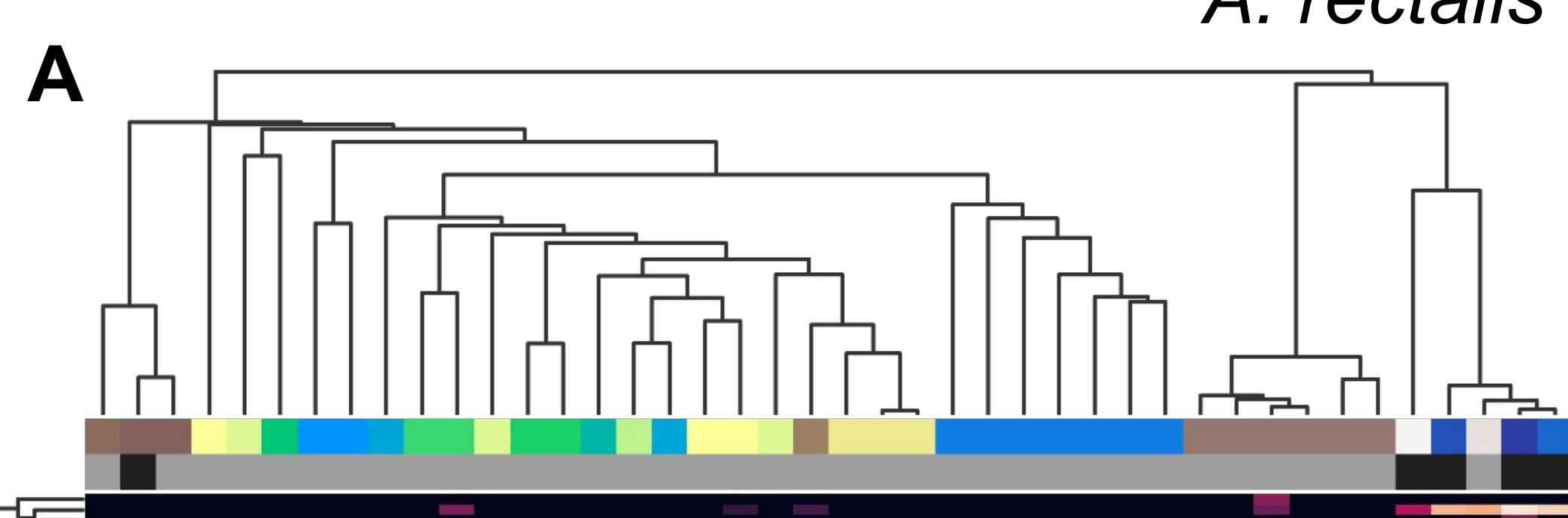

Westernized

Yes

No

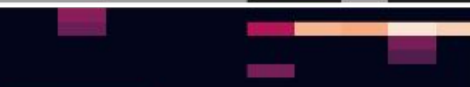

$+\sqrt{2}$

$+\sqrt{2}$
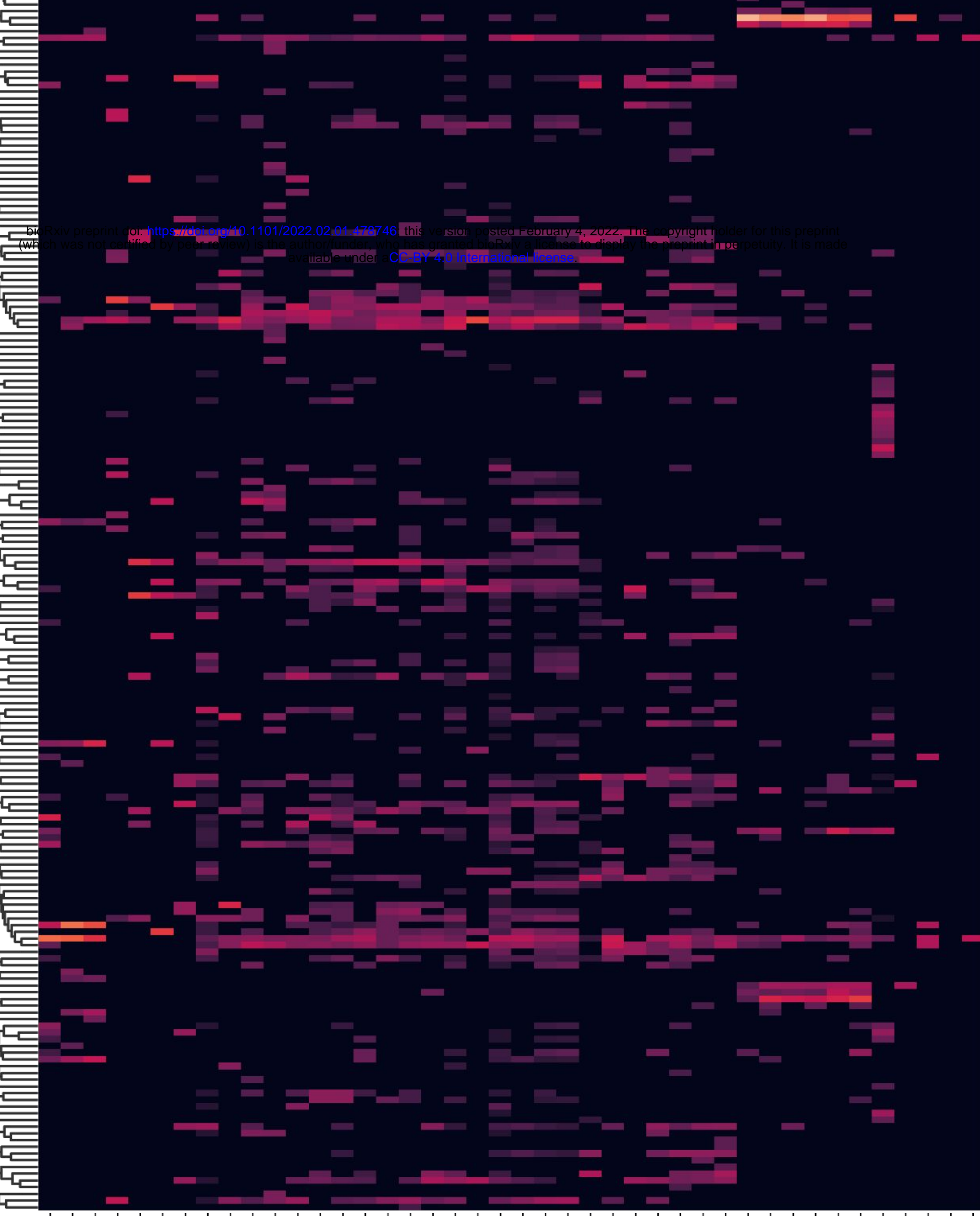

NovQJ

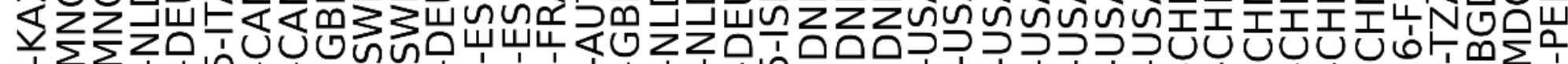

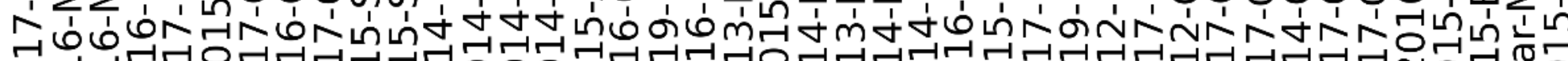

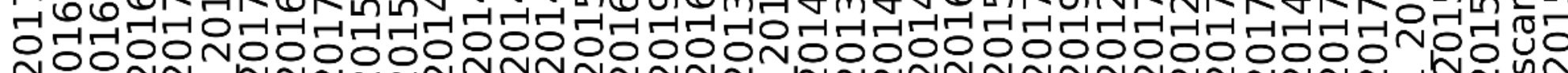
INN N N N N N N N N

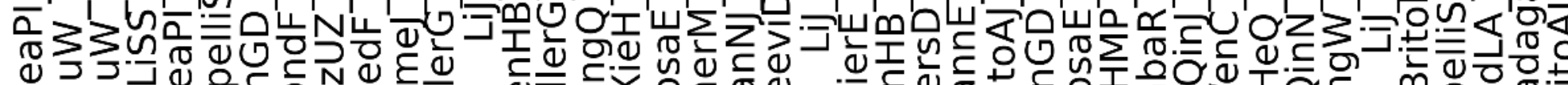

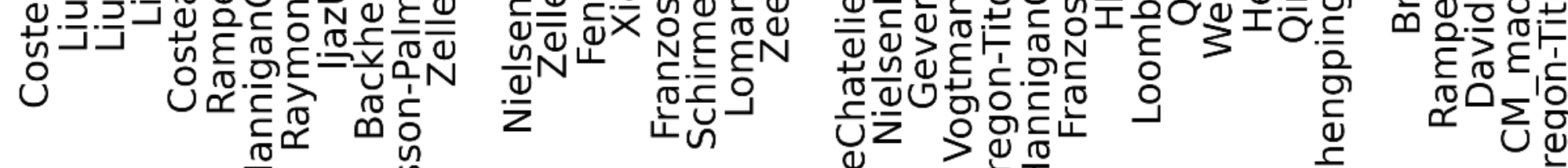

\section{Country}

$\begin{array}{lllll}\text { MDG } & \text { ITA } & \text { AUT } & \text { RUS } & \text { SGP } \\ \text { TZA } & \text { ESP } & \text { DEU } & \text { ISL } & \text { BRN } \\ \text { PER } & \text { SWE } & \text { NLD } & \text { ISR } & \text { IDN } \\ \text { USA } & \text { SVK } & \text { DNK } & \text { KAZ } & \text { MYS } \\ \text { CAN } & \text { HUN } & \text { NOR } & \text { MNG } & \text { BGD } \\ \text { GBR } & \text { EST } & \text { FIN } & \text { CHN } & \text { FJI } \\ \text { FRA } & & & & \end{array}$

B

M. smithii

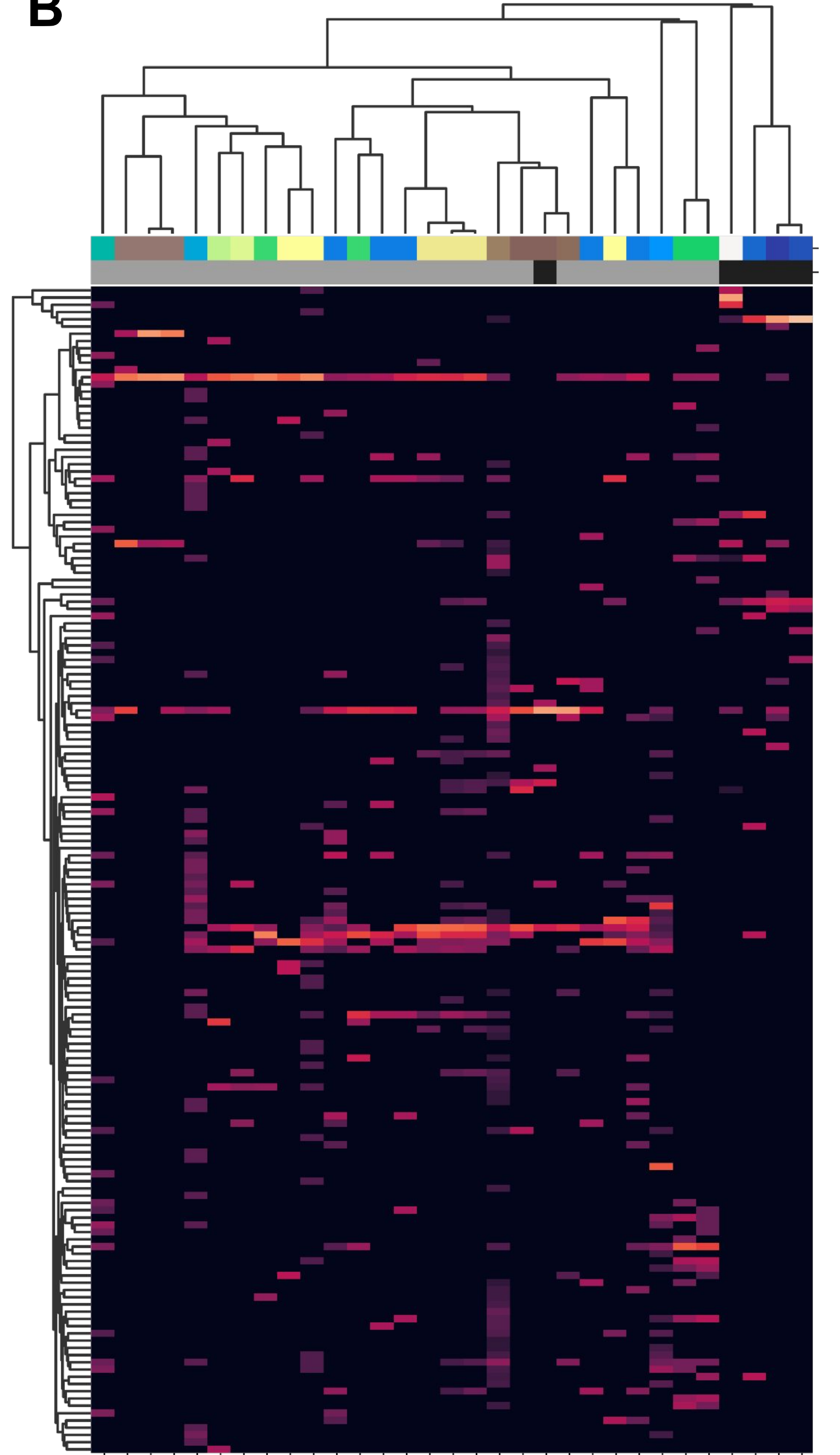

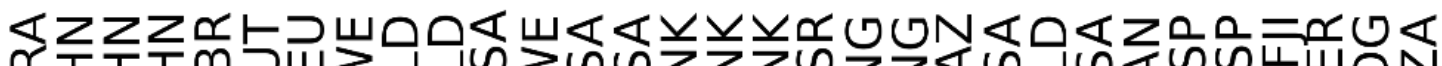

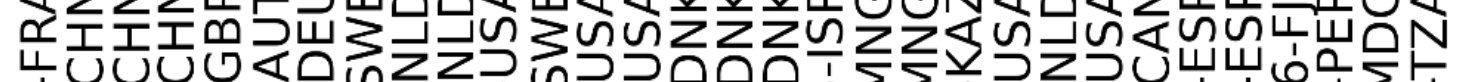

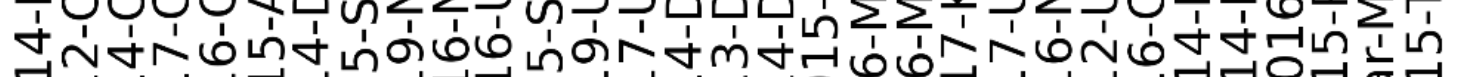

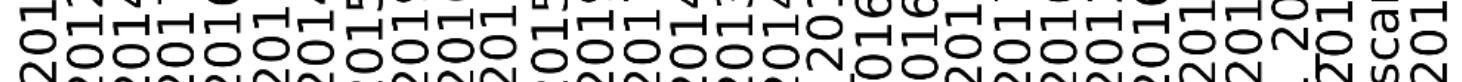
讨.

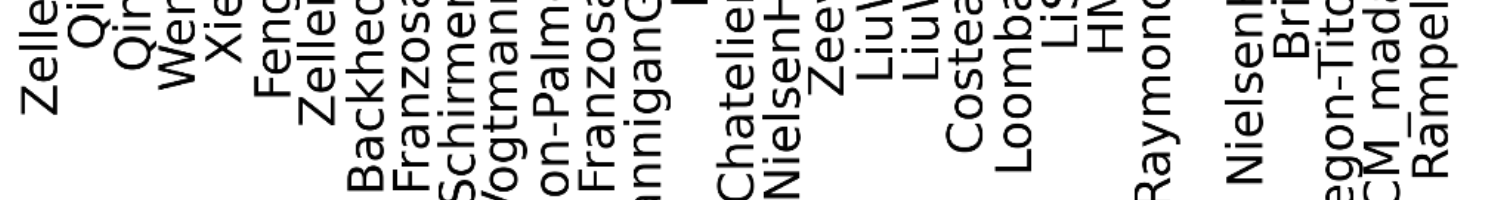




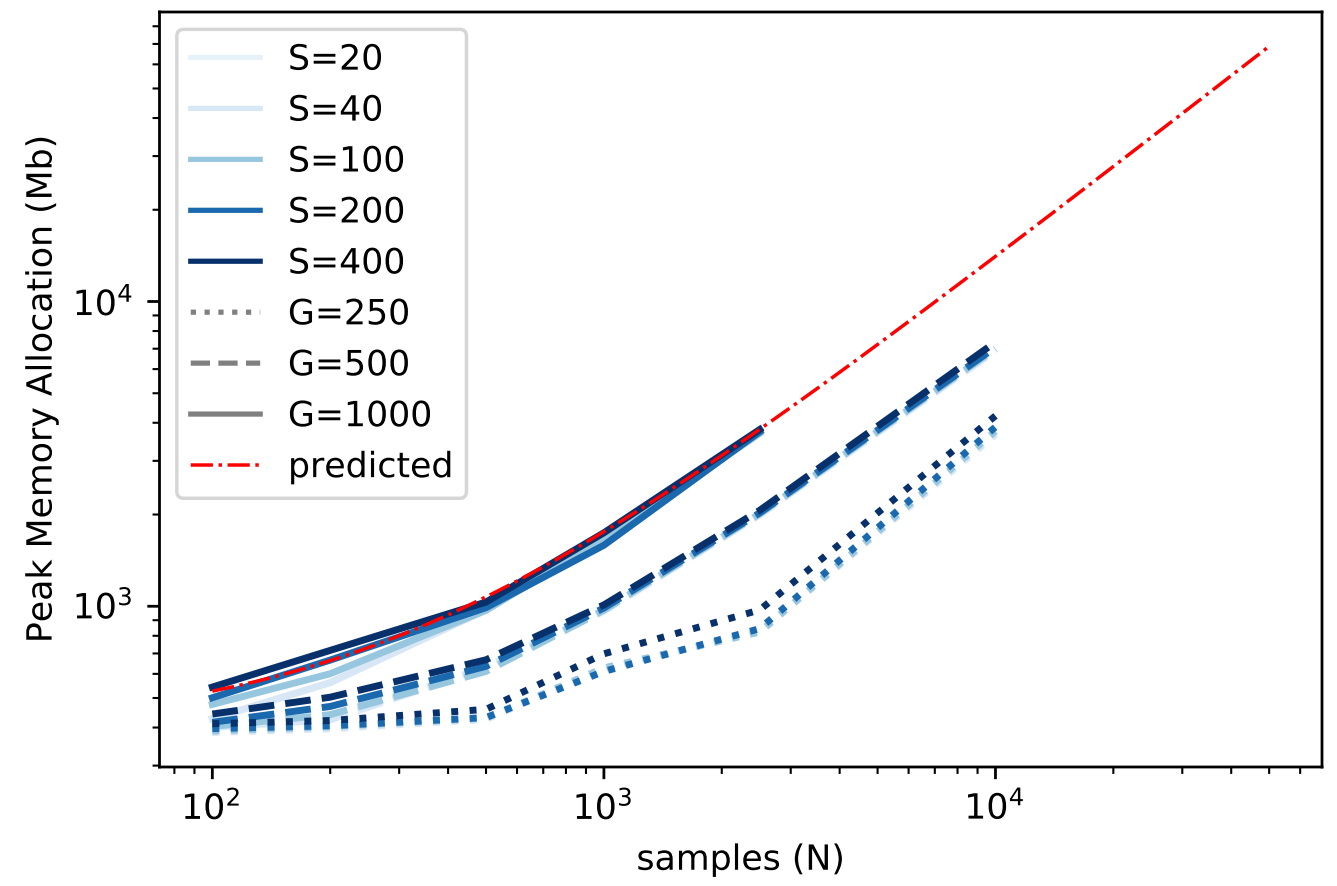




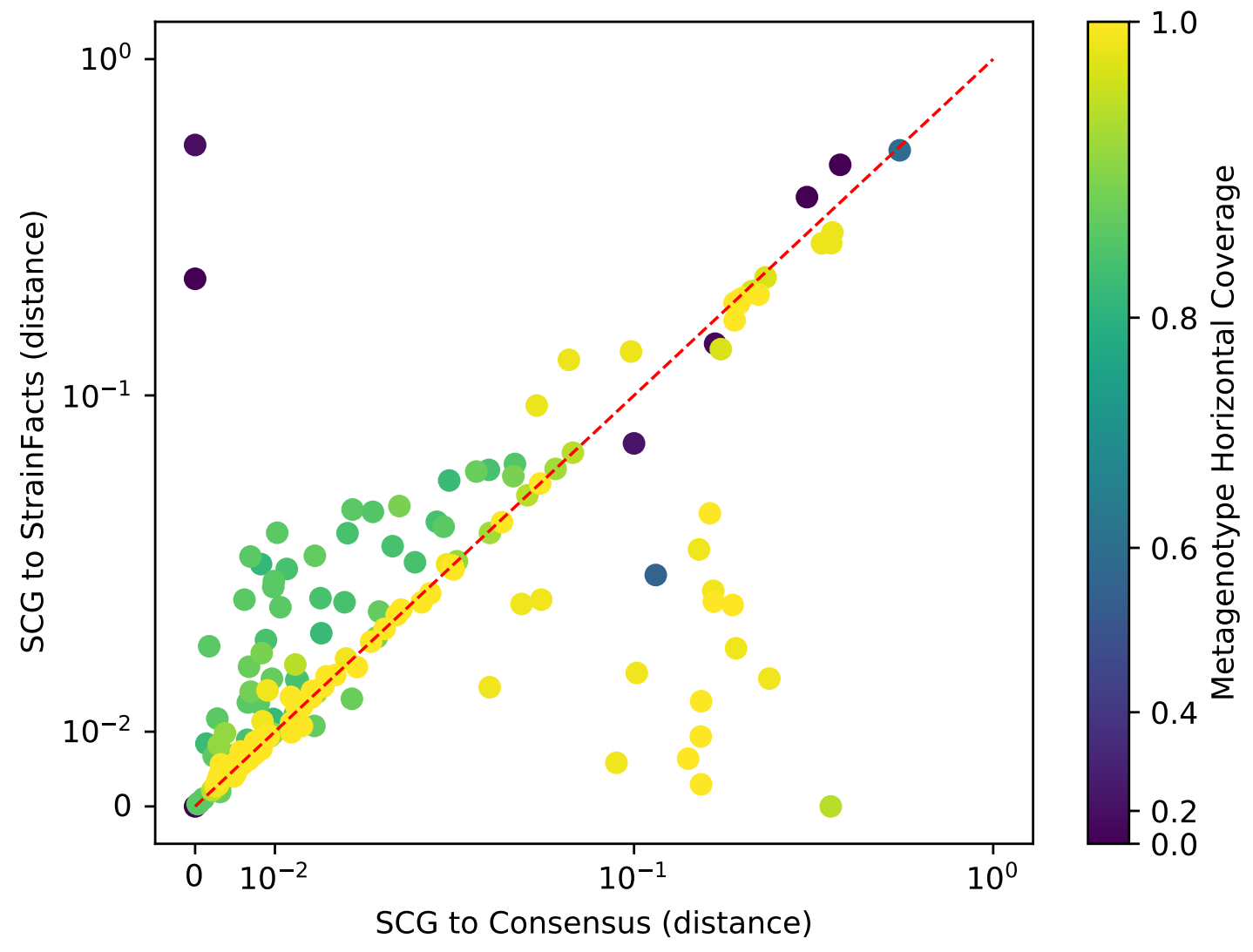




\section{Strain Dominance (frac. samples)}

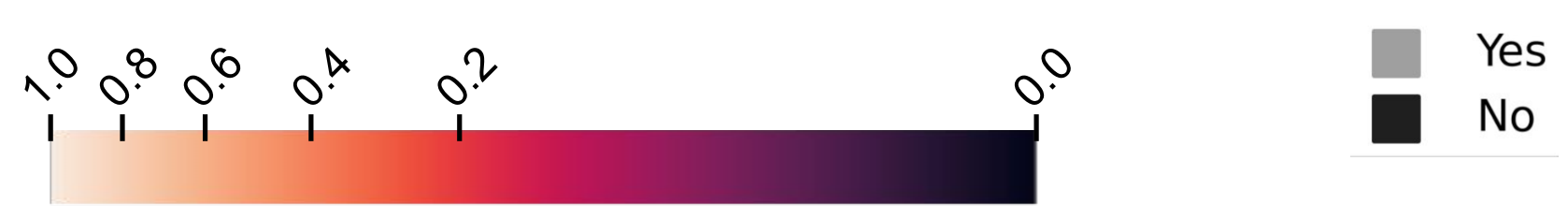

\section{Country}

\begin{tabular}{|l|l|l}
\hline MDG & ITA & AUT \\
TZA & ESP & DEU \\
PER & SWE & NLD \\
USA & SVK & DNK \\
CAN & HUN & NOR \\
GBR & EST & FIN \\
FRA & &
\end{tabular}

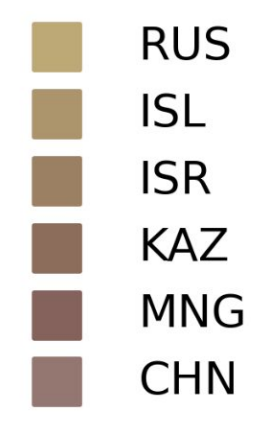

SGP

BRN

IDN

MYS

BGD

FJI

\section{E. coli}

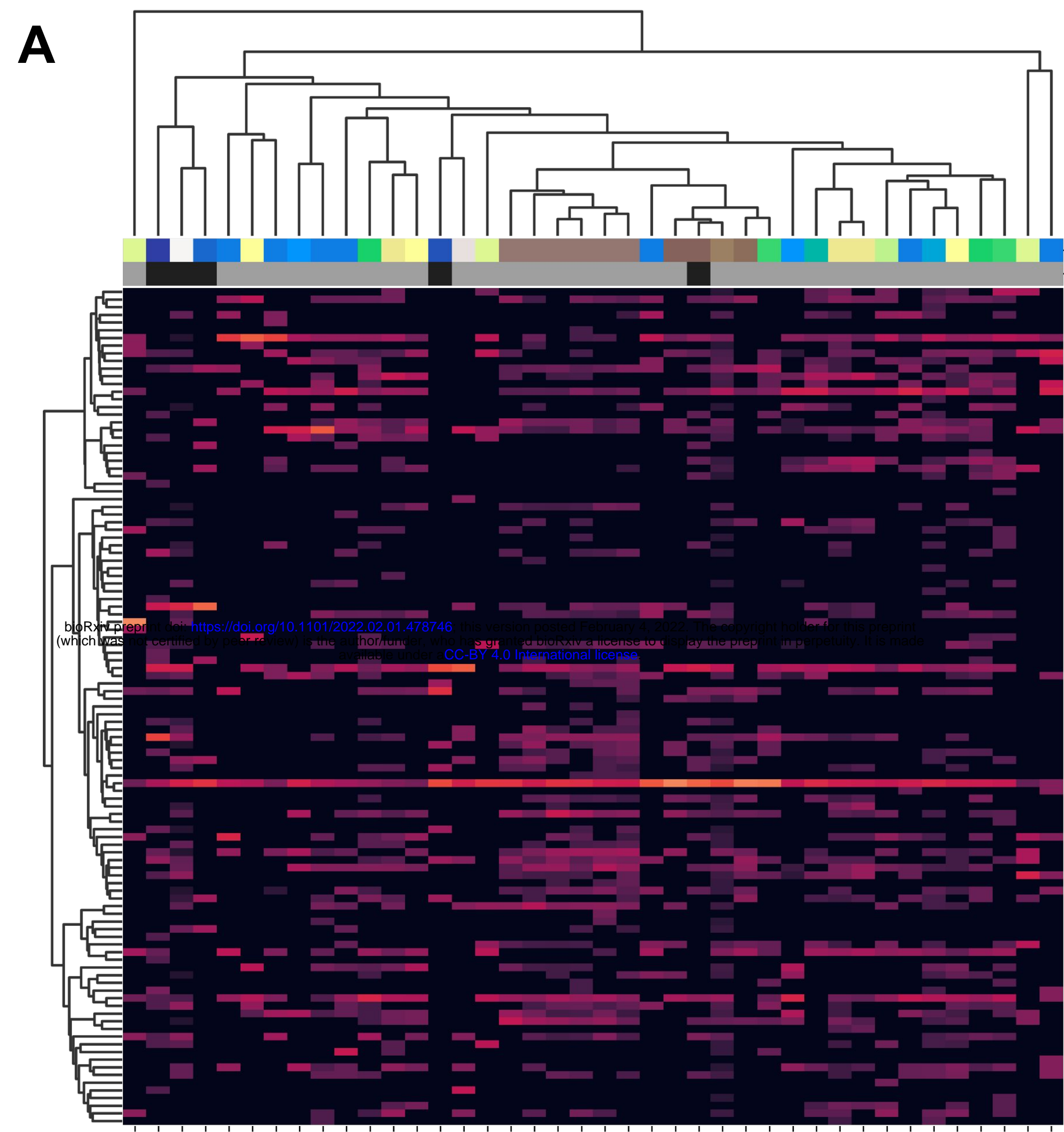

วण

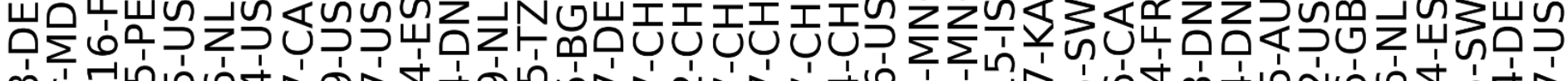
$m \frac{1}{0}$ 근

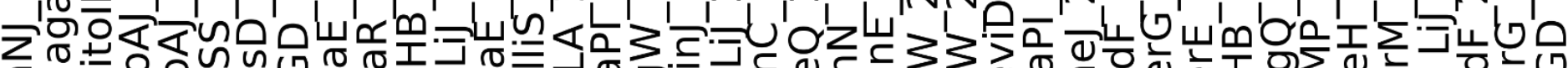

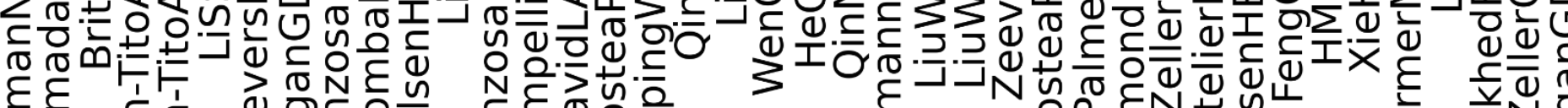

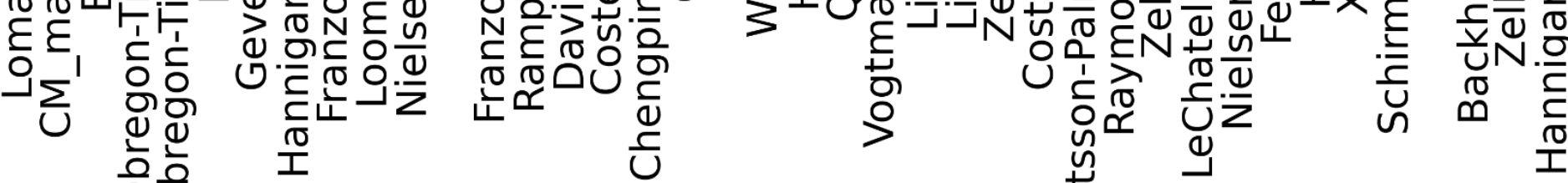

B

CAG-279

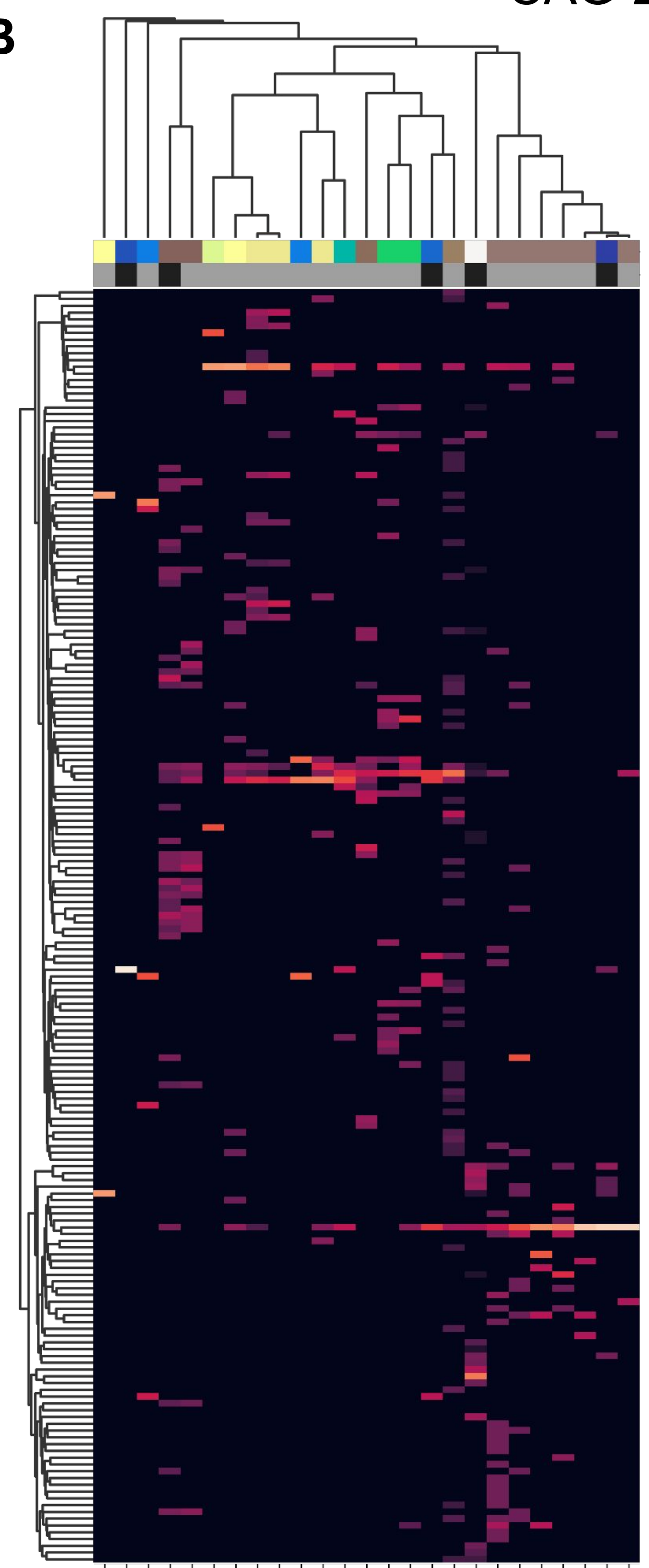

$0 \varangle \varangle 0030$ V

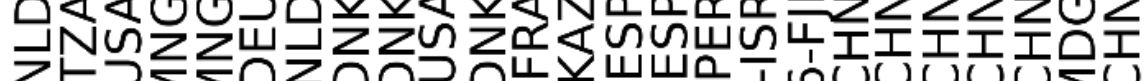

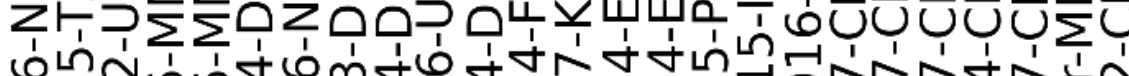

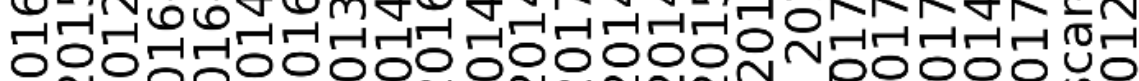

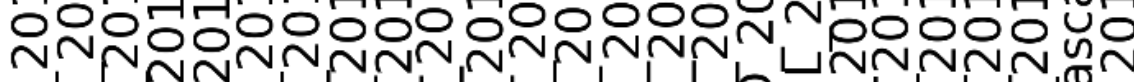

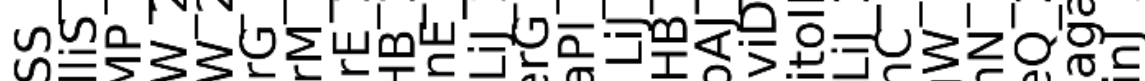

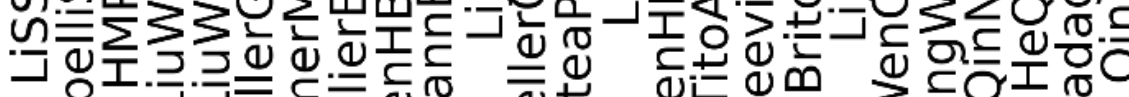

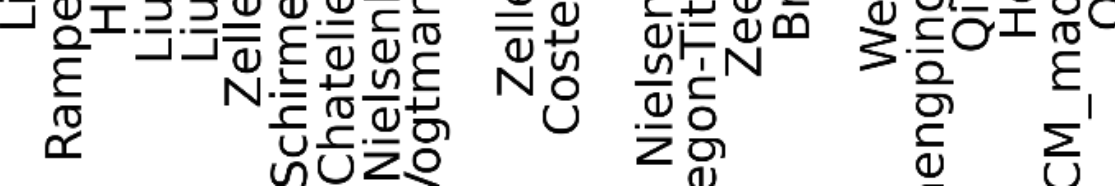

